\title{
The search for tolerant Lewis acid catalysts. Part 2: Enantiopure cycloalkyldialkylsilyl triflimide catalysts
}

\author{
Zilong Tang, ${ }^{\mathrm{a}, \mathrm{b}}$ Benoit Mathieu, ${ }^{\mathrm{a}}$ Bernard Tinant, ${ }^{\mathrm{a}}$ Georges Dive ${ }^{\mathrm{c}}$ and Léon Ghosez, ${ }^{\mathrm{a}, \mathrm{d}, *}$ \\ ${ }^{a}$ Department of Chemistry, Université catholique de Louvain, Place Louis Pasteur 1, B-1348 Louvain-la-Neuve, Belgium \\ ${ }^{\mathrm{b}}$ School of Chemistry and Chemical Engineering, Hunan University of Science and Technology, Xiangtan 411201, PR China \\ ${ }^{\mathrm{c}}$ Centre d'ingénierie des protéines, Institut de chimie B6, Université de Liège, 13, allée du 6 août Sart-Tilman, 4000 Liège, Belgium \\ ${ }^{\mathrm{d}}$ Institut Européen de Chimie et Biologie (IECB) rue Robert Escarpit, 2, F-33607 Pessac, France
}

Received 15 April 2007; accepted 22 May 2007

Available online 26 May 2007

\begin{abstract}
A series of 2-aryl- and arylmethyl-3-dialkylphenylsilyl cycloalkanones have been prepared and resolved. The pure enantiomers were reduced to the corresponding cycloalkane derivatives. These were used for the in situ generation of enantiopure cycloalkylsilyl triflimides by protodesilylation with bis(trifluoromethanesulfonyl)imide. The association of a bulky leaving group with a silicon atom carrying large substituents was again shown to favour the complexation of silicon with carbonyl groups: all these trialkylsilyl triflimides showed a high catalytic activity for Diels-Alder reactions. Enantiomeric excesses up to 59\% were observed. This is the highest enantioselectivity ever observed for a Diels-Alder reaction catalysed by a silicon Lewis acid.
\end{abstract}

(C) 2007 Elsevier Ltd. All rights reserved.

\section{Introduction}

In the course of our studies on enantioselective Diels-Alder reactions of activated azadienes, ${ }^{1}$ we found that many metalbased chiral Lewis acid catalysts could not be used as a result of competitive bindings to the diene and the dienophile carbonyl group. In the case of 2-azadienes a solution was found, which uses the bidentate imide group and the Box copper(II) catalyst developed by Evans and Johnson. ${ }^{2}$ However, this approach was totally unsuccessful for 1-azadiene cycloadditions. ${ }^{3}$ Another solution for efficient catalysis of 2-azadienes cycloadditions was the use of a more basic activating group of the dienophilic double bond in conjunction with silyltriflates as Lewis acid catalysts: $\alpha, \beta$-unsaturated amides were shown to react smoothly with 2 -azadienes in the presence of TBDMSOTf. ${ }^{4}$ However, silyltriflates were not acidic enough to activate the corresponding esters. In 1997, both our group and Mikami's group discovered that $N$-(trimethylsilyl)triflimide $\left(\mathrm{Me}_{3} \mathrm{SiNTf}_{2}\right)$ was an extremely efficient carbonyl activator. ${ }^{5,6}$ We observed an unexpected reversal of thermodynamic acidity on going from the Brönsted acids $\left(\mathrm{HOTf}>\mathrm{HNTf}_{2}\right)$ to the TMS derivatives (TMSOTf $<$ TMSNTf $_{2}$ ), which was assigned to the larger size of the triflimide group as compared to triflate. ${ }^{5}$ Also our group demonstrated that increasing the size of the alkyl substituents on silicon resulted in a significant increase of Lewis acidity. ${ }^{7}$ It was later shown by several groups that $\mathrm{TMSNTf}_{2}$, often

Keywords: Lewis acid; Enantioselective catalysis; Asymmetric Diels-Alder cycloadditions; Silylated triflimides.

* Corresponding author. E-mail: ghosez@chim.ucl.ac.be generated in situ, was an efficient catalyst for many carboncarbon bond-forming reactions, ${ }^{8}$ or, e.g., the acid-catalysed ring-opening polymerisation of cyclosiloxanes. ${ }^{9}$

Stimulated by these successful results, we decided to examine the possibility of generating chiral silyl triflimides, which could act as Lewis acids for the activation of carbonyl groups. The possibility of using an asymmetric silicon atom was rejected since these compounds are not readily accessible and steric course of substitution at silicon is complex. At the outcome of these studies, no such catalyst had been described. Examples of Diels-Alder reactions catalysed by an enantiopure silicon catalyst were described in 1998 but facial selectivities were very modest $(e e \leq 10 \%) .{ }^{10}$ More recently Leighton et al. have reported the first examples of highly enantioselective carbon-carbon bond-forming reactions catalysed by silyl chlorides derived from enantiopure aminoalcohols. ${ }^{11}$ At this point, however, the scope and limitations of this elegant approach have not yet been defined.

Our approach was inspired by the successful studies of chiral boron Lewis acid catalysts by Hawkins' group. ${ }^{12}$ We first prepared several silylated triflimides derived from (-)myrtenal and tested them as catalysts of the Diels-Alder reactions of methyl acrylate and methyl crotonate to cyclopentadiene (Scheme 1). ${ }^{7}$ The best ee was $\approx 54 \%$. We now wish to report the details of our studies of a variety of $\alpha$-aryl- or arylmethyl-cycloalkylsilyl triflimides as catalysts for the asymmetric Diels-Alder reactions of $\alpha, \beta$-unsaturated esters. 


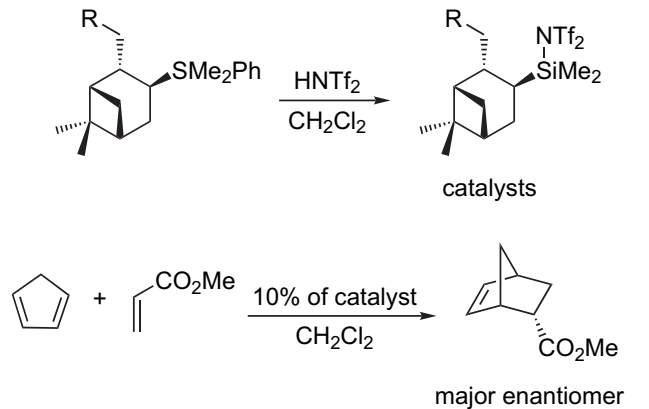

Scheme 1. Silylated triflimides derived from (-)-myrtenal. For $\mathrm{R}=\mathrm{OMe}$ at $-78{ }^{\circ} \mathrm{C}$, yield $=83 \%$, ee $=54 \%$.

\section{Synthesis of the enantiopure scaffolds}

\subsection{Synthesis of trans-2-aryl-3-dialkylphenylsilyl cyclo- alkanones}

2,3-Epoxyketones are versatile building blocks in organic synthesis. ${ }^{13}$ They have been shown to behave as versatile equivalents of $\alpha$-ketovinyl cations. ${ }^{14}$ Compounds 1a-c were prepared by epoxidation of the cyclic enones with hydrogen peroxide under basic conditions following literature procedures $^{15}$ (Scheme 2).

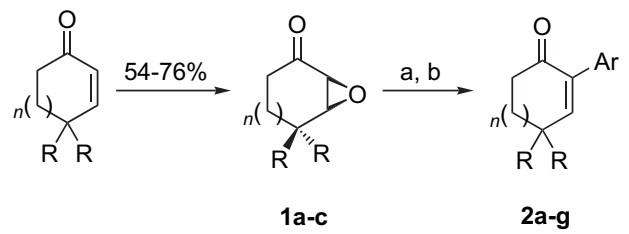

Scheme 2. Reagents and conditions: (a) LDA (1.1 equiv) in THF, $-78^{\circ} \mathrm{C}$; (b) ArLi (2 equiv), $-23{ }^{\circ} \mathrm{C}$ then PTSA (cat) in refluxing toluene.

$\boldsymbol{\alpha}$-Aryl enones $\mathbf{2 a}-\mathbf{g}$ were prepared following Wender's general procedure ${ }^{14 \mathrm{~d}}$ (Scheme 2, Table 1). Prior formation of an enolate by treatment of 1a-c with LDA was essential to ensure the chemo- and regioselective opening of the epoxide ring by the aryl lithium reagent.

The reaction of $\mathrm{CuCN}$ with dimethyl- or diethylphenylsilyllithium generated the corresponding cuprate reagents, which were reacted with enones $\mathbf{2 a -}-\mathbf{g}$ according to Fleming's procedure (Scheme 3, Table 2) ${ }^{18}$ The reactions were quenched by methanol at room temperature: these conditions only gave the trans-adduct $\mathbf{3 a}-\mathbf{h}$ as predicted on the basis of the early observations of the group of Seyden-Penne. ${ }^{19}$ The trans-stereoselectivity was probably the result of a thermodynamically controlled protonation occurring at higher temperature and in more basic conditions. ${ }^{19}$ Indeed, quench with a saturated $\mathrm{NH}_{4} \mathrm{Cl}$ solution at $0{ }^{\circ} \mathrm{C}$ mainly gave mixtures of stereoisomers (cis-4 $>$ trans-3) except when $n=0$ and $\mathrm{Ar}=1-\mathrm{Np}$ (only trans).

Figure 1 shows the typical ${ }^{1} \mathrm{H}$ and ${ }^{13} \mathrm{C}$ NMR patterns and assignments for a cis-adduct 4a. The cis- and trans-isomers were distinguished by the values of coupling constants $J_{\mathrm{H}_{2}-\mathrm{H}_{3}}$, which varied from 5.1 to $5.7 \mathrm{~Hz}$ for the $c i s$-isomers
Table 1. $\alpha$-Arylation of epoxyketones 1a-c

(lit)

Scheme 3. Reagents and conditions: (a) $\mathrm{PhMe}_{2} \mathrm{SiCl}$ or $\mathrm{PhEt}_{2} \mathrm{SiCl}$ (3 equiv), $\mathrm{Li}$ (9 equiv), THF; (b) addition of $\mathrm{PhR}_{2}^{\prime} \mathrm{SiLi}$ in suspension of $\mathrm{CuCN}$ (1.5 equiv), $-30{ }^{\circ} \mathrm{C}$ then $0{ }^{\circ} \mathrm{C}$; (c) $2 \mathbf{2 a}-\mathbf{g}$ ( 1 equiv), THF, $-23^{\circ} \mathrm{C} \rightarrow \mathrm{rt}$ then quench.

and from 11.1 to $13.0 \mathrm{~Hz}$ for the trans-isomers. ${ }^{20}$ As shown later, the structure and configuration of $\mathbf{3 f}$ was confirmed by $\mathrm{X}$-ray diffraction analysis.

Table 2. Michael additions of phenyldialkylsilyl cuprate reagents to 2-arylcycloalkenones

\begin{tabular}{|c|c|c|c|c|c|c|c|}
\hline \multirow{2}{*}{\multicolumn{2}{|c|}{ Method of quenching ${ }^{\mathrm{a}}$}} & \multirow[t]{2}{*}{$n$} & \multirow[t]{2}{*}{$\mathrm{R}$} & \multirow[t]{2}{*}{$\mathrm{R}^{\prime}$} & \multirow[t]{2}{*}{$\mathrm{Ar}$} & \multicolumn{2}{|c|}{ Yield, $^{\mathrm{b}} \%$} \\
\hline & & & & & & 3 (trans) & $4(c i s)$ \\
\hline a & A & 1 & $\mathrm{H}$ & $\mathrm{Me}$ & $\mathrm{Ph}$ & 3 & 70 \\
\hline $\mathrm{a}$ & B & 1 & $\mathrm{H}$ & $\mathrm{Me}$ & $\mathrm{Ph}$ & 71 & - \\
\hline $\mathrm{b}$ & A & 1 & $\mathrm{H}$ & $\mathrm{Me}$ & $1-\mathrm{Np}$ & 34 & 23 \\
\hline $\mathrm{c}$ & B & 1 & $\mathrm{H}$ & $\mathrm{Me}$ & 9-An & 20 & - \\
\hline $\mathrm{c}$ & A & 1 & $\mathrm{H}$ & $\mathrm{Me}$ & 9-An & - & 15 \\
\hline d & $\mathrm{B}$ & 1 & $\mathrm{H}$ & $\mathrm{Me}$ & 2-PhOMe & 67 & - \\
\hline $\mathrm{e}$ & B & 1 & $\mathrm{H}$ & $\mathrm{Me}$ & 2-PhOPh & 57 & - \\
\hline $\mathrm{f}$ & B & 1 & $\mathrm{Me}$ & $\mathrm{Me}$ & $1-\mathrm{Np}$ & 50 & - \\
\hline $\mathrm{g}$ & A & 0 & $\mathrm{H}$ & $\mathrm{Me}$ & $1-\mathrm{Np}$ & 73 & - \\
\hline $\mathrm{h}$ & B & 1 & $\mathrm{H}$ & Et & $\mathrm{Ph}$ & 70 & - \\
\hline
\end{tabular}

a A: aqueous $\mathrm{NH}_{4} \mathrm{Cl}, 0^{\circ} \mathrm{C}$; B: $\mathrm{MeOH}, \mathrm{rt}$.

${ }^{b}$ Yields of isolated products. 


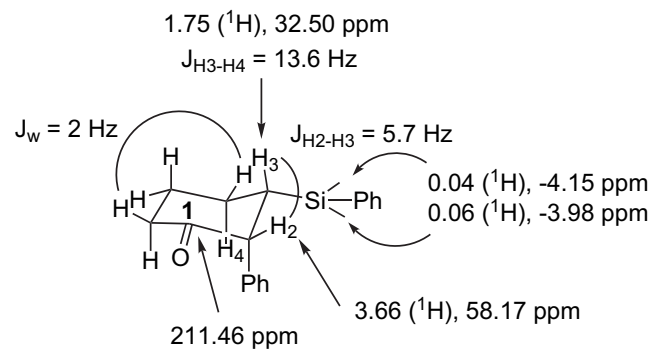

Figure 1. Typical ${ }^{1} \mathrm{H}$ and ${ }^{13} \mathrm{C}$ NMR patterns for a cis Michael adduct $4 \mathbf{a}$.

\subsection{Synthesis of trans-2-arylmethyl-3-dimethylphenyl- silyl-cyclohexanones 5}

A series of trans-2-arylmethyl-3-silyl cycloalkanones 5a-c were prepared by tandem reactions involving the 1,4-addition of silyl cuprate to cyclohexenone followed by reaction of the in situ generated enolates with arylmethyl bromides (Scheme 4, Table 3). ${ }^{21}$ In all cases we obtained the pure trans-2-arylmethyl-3-silyl cycloalkanones 5 after column chromatography. In most cases, small amounts of diarylmethylated products 6 were also formed. However, we were not able to avoid the formation of $\mathbf{6}$ without significantly lowering the yield of $\mathbf{5}$. In all cases the reactions were highly trans-stereoselective.

\subsection{Resolution of the racemic cycloalkanones}

Racemic ketones 3a-h and 5a-c were resolved by preparative HPLC. Chiralcell AD or OD-H columns gave excellent separation of these enantiomeric ketones. Enantiomers eluting first showed negative optical rotations while the isomers with longer retention times showed positive optical rotations. The absolute configurations of enantiomerically pure trans-cycloalkanone (-)-3f was shown to be $(2 R, 3 S)$ by X-ray diffraction analysis (Fig. 2) while (+)-5a and $(+)-5 \mathbf{b}$ were shown to be the $(2 S, 3 S)$ configurations (Figs. 3 and 4). ${ }^{22}$ The absolute configuration of other transcycloalkanones could be inferred from the above results: the enantiomeric ketones with positive optical rotations were assigned absolute configuration $(2 S, 3 S)$ and those with negative optical rotations were assigned configuration $(2 R, 3 R)$.

\subsection{Synthesis of ENP 2-dialkylphenylsilyl-aryl (arylmethyl)-cycloalkanes}

The carbonyl group of enantiomerically pure (ENP) cycloalkanones was reduced following the experimental protocol described by Kim et al. ${ }^{23}$ The ketone was first converted into the corresponding tosylhydrazone. The crude tosylhydrazones showed two spots on TLC suggesting the presence
Table 3. Tandem 1,4-addition-alkylation of cyclohexenone and cyclopentenone

\begin{tabular}{lllll}
\hline & Ar & $n$ & $\begin{array}{l}\text { Yields } \\
\text { of } \mathbf{5}, \%\end{array}$ & $\begin{array}{l}\text { Yields } \\
\text { of } \mathbf{6}, \%\end{array}$ \\
\hline a & $\mathrm{Ph}$ & 1 & 85 & 9 \\
b & $2-\mathrm{Np}$ & 1 & 41 & 4 \\
c & $1-\mathrm{Np}$ & 1 & 39 & 7 \\
\hline
\end{tabular}

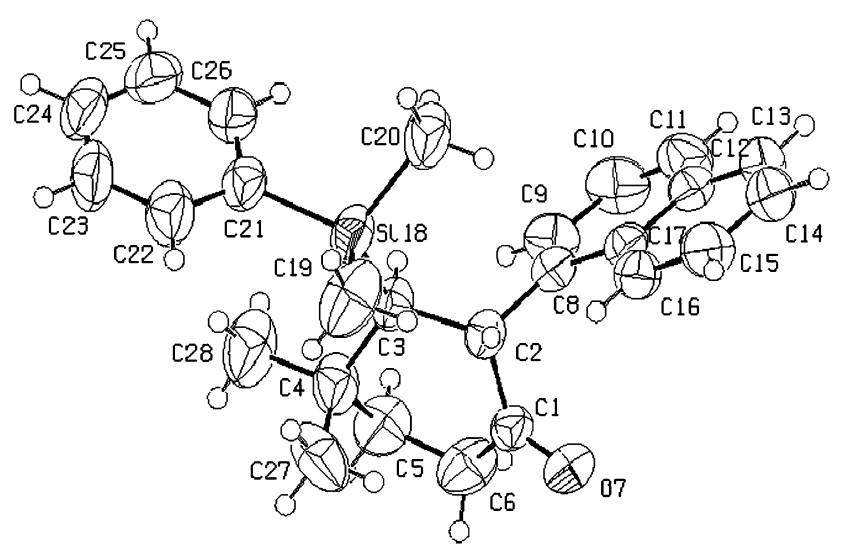

Figure 2. ORTEP drawing of compound (-)-3f.

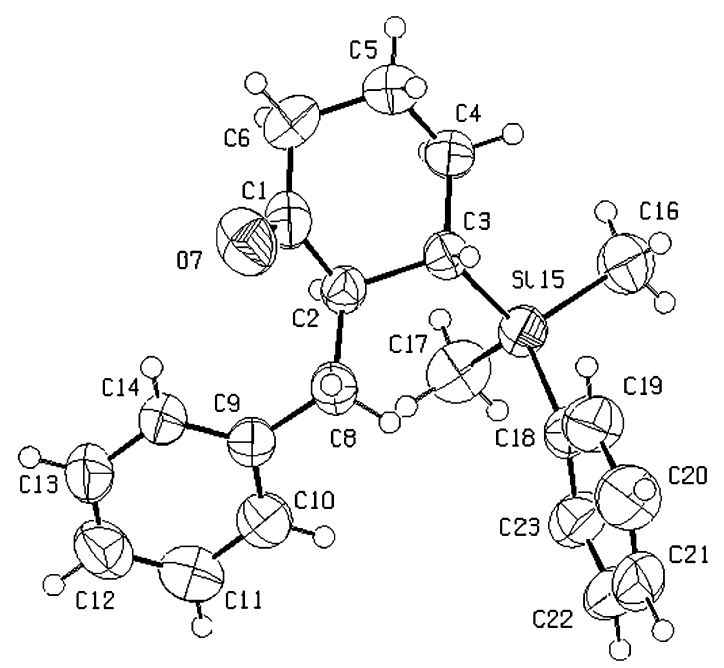

Figure 3. ORTEP drawing of compound (+)-5a.

of syn and anti isomers. Reduction of the crude hydrazone with $\mathrm{NaBH}_{3} \mathrm{CN} / \mathrm{ZnCl}_{2}$ (2:1) yielded the corresponding cycloalkane (Scheme 5, Table 4). ${ }^{23}$ Yields were moderate to good except for the reduction of the tosylhydrazone derived from ketone 3c, which gave a complex mixture of products.
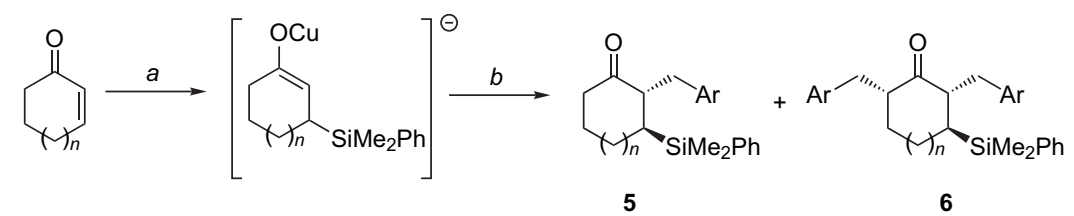

Scheme 4. Reagents and conditions: (a) $\mathrm{PhMe} 2 \mathrm{SiLi}$ ( 3 equiv), $\mathrm{CuI}\left(1.5\right.$ equiv), in THF/HMPA, $-30{ }^{\circ} \mathrm{C}$ then addition of enone $\left(1\right.$ equiv) at $-78^{\circ} \mathrm{C} \rightarrow-23^{\circ} \mathrm{C}$; (b) arylmethyl bromide (2.8 equiv), $-78^{\circ} \mathrm{C}$ then aqueous $\mathrm{NH}_{4} \mathrm{Cl}$ at $0{ }^{\circ} \mathrm{C}$. 


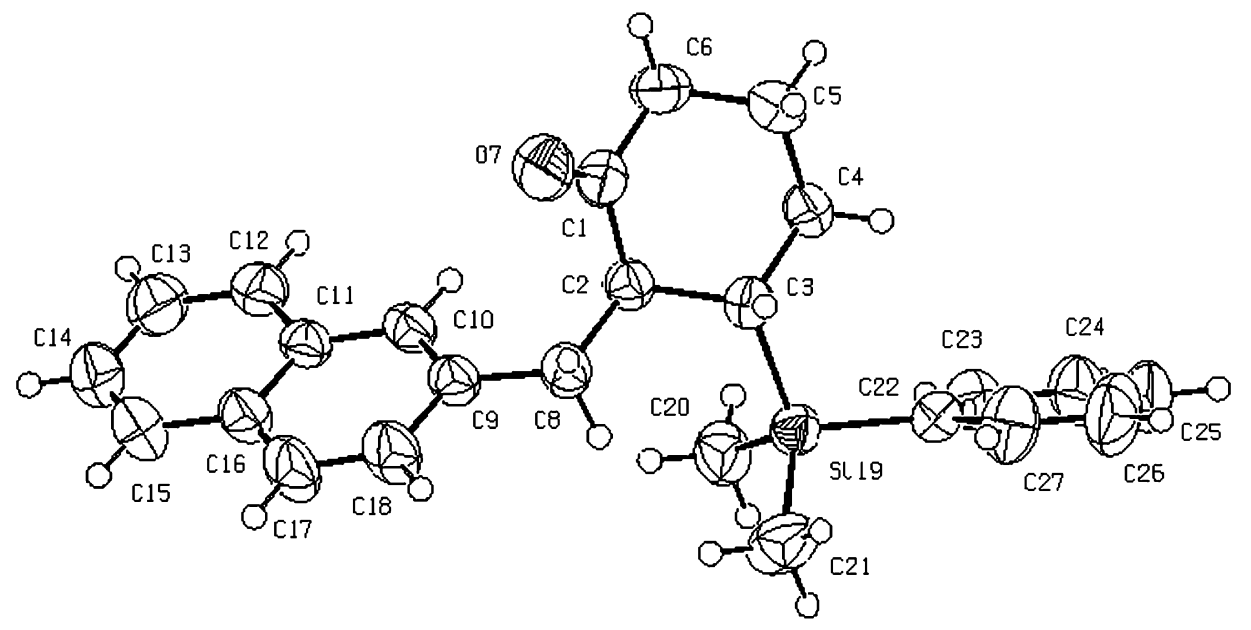

Figure 4. ORTEP drawing of compound (+)-5b

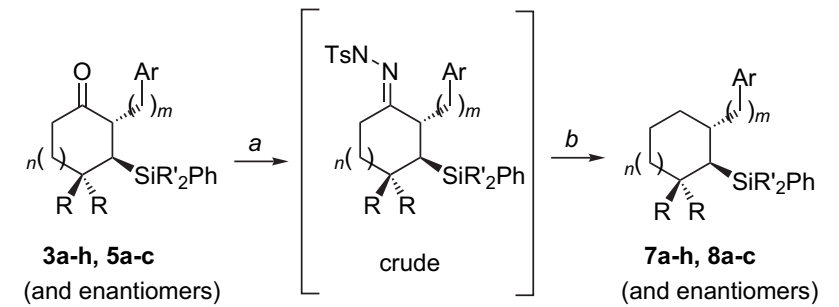

Scheme 5. Reagents and conditions: (a) $\mathrm{TsNHNH}_{2}$ (1.2 equiv), EtOH, $60{ }^{\circ} \mathrm{C}, 3-6 \mathrm{~h}$; (b) $\mathrm{NaBH}_{3} \mathrm{CN}$ (5 equiv), $\mathrm{ZnCl}_{2}$ (2.5 equiv), $\mathrm{EtOH} / \mathrm{MeOH}$ (1:1), $60{ }^{\circ} \mathrm{C}, 3-10 \mathrm{~h}$.

When applied to the cis-isomers $\mathbf{4 a}$, this method gave a mixture of cis- and trans-1,2-disubstituted cycloalkanes: epimerisation probably occurred during the formation of the tosylhydrazone. We then turned to an alternative reductive method involving the formation of a dithioketal followed by a desulfurisation reaction with Raney nickel (Scheme 6). ${ }^{24,25}$

Table 4. Reduction of ENP 3-silylated cycloalkanones

\begin{tabular}{|c|c|c|c|c|c|c|c|}
\hline Ketone & $n$ & $\mathrm{~m}$ & $\mathrm{R}$ & $\mathrm{R}^{\prime}$ & $\mathrm{Ar}$ & Product & $\begin{array}{l}\text { Yield, }{ }_{\%}^{\mathrm{a}}\end{array}$ \\
\hline$(+)-\mathbf{3 a}$ & 1 & 0 & $\mathrm{H}$ & $\mathrm{Me}$ & $\mathrm{Ph}$ & $(-)-7 \mathbf{a}$ & 31 \\
\hline$(-)-\mathbf{3 a}$ & 1 & 0 & $\mathrm{H}$ & $\mathrm{Me}$ & $\mathrm{Ph}$ & $(+)-7 \mathbf{a}$ & 44 \\
\hline$(+)-3 \mathbf{b}$ & 1 & 0 & $\mathrm{H}$ & $\mathrm{Me}$ & $1-\mathrm{Np}$ & $(+)-7 \mathbf{b}$ & 45 \\
\hline$(-)-3 \mathbf{b}$ & 1 & 0 & $\mathrm{H}$ & $\mathrm{Me}$ & $1-\mathrm{Np}$ & $(-)-7 \mathbf{b}$ & 39 \\
\hline$(+)-3 c$ & 1 & 0 & $\mathrm{H}$ & $\mathrm{Me}$ & 9-An & \multicolumn{2}{|c|}{ Complex mixture } \\
\hline$(+)-\mathbf{3 d}$ & 1 & 0 & $\mathrm{H}$ & $\mathrm{Me}$ & 2-PhOMe & $(-)-7 d$ & 51 \\
\hline$(-)-3 d$ & 1 & 0 & $\mathrm{H}$ & $\mathrm{Me}$ & 2-PhOMe & $(+)-7 d$ & 57 \\
\hline$(+)-\mathbf{3 e}$ & 1 & 0 & $\mathrm{H}$ & $\mathrm{Me}$ & 2-PhOPh & $(+)-7 \mathbf{e}$ & 20 \\
\hline$(-)-\mathbf{3 e}$ & 1 & 0 & $\mathrm{H}$ & $\mathrm{Me}$ & 2-PhOPh & $(-)-7 \mathrm{e}$ & 25 \\
\hline$(+)-\mathbf{3 f}$ & 1 & 0 & $\mathrm{Me}$ & $\mathrm{Me}$ & $1-\mathrm{Np}$ & $(+)-7 f$ & 31 \\
\hline$(-)-3 f$ & 1 & 0 & $\mathrm{Me}$ & $\mathrm{Me}$ & $1-\mathrm{Np}$ & $(-)-7 \mathbf{f}$ & 27 \\
\hline$(+)-3 g$ & 0 & 0 & $\mathrm{H}$ & $\mathrm{Me}$ & $1-\mathrm{Np}$ & $(+)-7 \mathbf{g}$ & 32 \\
\hline$(-)-3 g$ & 0 & 0 & $\mathrm{H}$ & $\mathrm{Me}$ & $1-\mathrm{Np}$ & $(-)-7 g$ & 32 \\
\hline$(+)-3 h$ & 1 & 0 & $\mathrm{H}$ & Et & $\mathrm{Ph}$ & $(-)-7 \mathrm{~h}$ & 31 \\
\hline$(-)-3 \mathbf{h}$ & 1 & 0 & $\mathrm{H}$ & Et & $\mathrm{Ph}$ & $(+)-7 \mathbf{h}$ & 35 \\
\hline$(+)-5 \mathbf{a}$ & 1 & 1 & $\mathrm{H}$ & $\mathrm{Me}$ & $\mathrm{Ph}$ & $(+)-8 \mathbf{a}$ & 54 \\
\hline$(-)-5 \mathbf{a}$ & 1 & 1 & $\mathrm{H}$ & $\mathrm{Me}$ & $\mathrm{Ph}$ & $(-)-\mathbf{8 a}$ & 85 \\
\hline$(+)-5 \mathbf{b}$ & 1 & 1 & $\mathrm{H}$ & $\mathrm{Me}$ & $2-\mathrm{Np}$ & $(+)-\mathbf{8 b}$ & 34 \\
\hline$(-)-5 \mathbf{b}$ & 1 & 1 & $\mathrm{H}$ & $\mathrm{Me}$ & $2-\mathrm{Np}$ & $(-)-8 \mathbf{b}$ & 38 \\
\hline$(+)-5 c$ & 1 & 1 & $\mathrm{H}$ & $\mathrm{Me}$ & $1-\mathrm{Np}$ & $(+)-8 c$ & 31 \\
\hline$(-)-5 c$ & 1 & 1 & $\mathrm{H}$ & $\mathrm{Me}$ & $1-\mathrm{Np}$ & $(-)-8 c$ & 33 \\
\hline
\end{tabular}

${ }^{\mathrm{a}}$ Yields based on ketone for the two-step sequence.

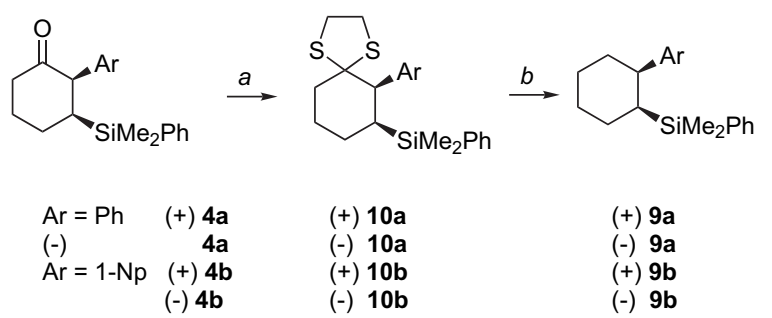

Scheme 6. Reagents and conditions: (a) ketone (1 equiv), ethanedithiol (1.2 equiv), $\mathrm{TiCl}_{4}$ (0.3 equiv) in $\mathrm{CH}_{2} \mathrm{Cl}_{2},-40{ }^{\circ} \mathrm{C} \rightarrow \mathrm{rt}$; (b) $10 \mathrm{a}$ or $10 \mathrm{~b}$ ( 1 equiv), $\mathrm{Ra}-\mathrm{Ni}$ (10 equiv) in $\mathrm{CH}_{3} \mathrm{OH}$, rt.

\section{Synthesis of the ENP silylated triflimides}

In an earlier paper in this series, we showed that $\mathrm{TMSNTf}_{2}$ could be quantitatively generated by protodesilylation of the corresponding phenyltrimethylsilane. ${ }^{18 c, 26}$ We have applied this method for the preparation of the new ENP catalysts (Scheme 7). The transformation of phenylsilanes into the corresponding silylated triflimides was monitored by ${ }^{1} \mathrm{H}$ NMR. The signals of the protons of the methyl groups connected to the silicon atom moved downfield (from 0.20 to $0.60 \mathrm{ppm}$ ) and a signal appeared at $7.36 \mathrm{ppm}$ for the protons of benzene. When silicon carries two ethyl groups, the protodesilylation reaction became very slow at room temperature $\left(\sim 10 \%\right.$ conversion after $3 \mathrm{~h}$ in $\left.\mathrm{CH}_{2} \mathrm{Cl}_{2}\right)$ but was quantitative at $80{ }^{\circ} \mathrm{C}$ in toluene (Scheme 8).

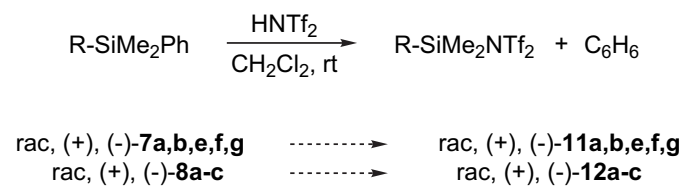

Scheme 7. General procedure for the generation of phenyldialkylsilyltriflimide catalysts.

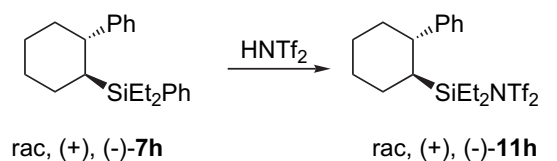

Scheme 8. Reagents and conditions: $\mathrm{CH}_{2} \mathrm{Cl}_{2}, \mathrm{rt}, 3 \mathrm{~h}$, yield $=10 \%$ or toluene, $80{ }^{\circ} \mathrm{C}, 3 \mathrm{~h}, \sim 100 \%$. 


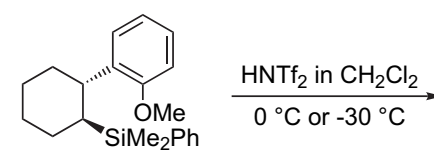

$(-)-7 d$

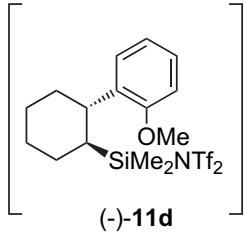

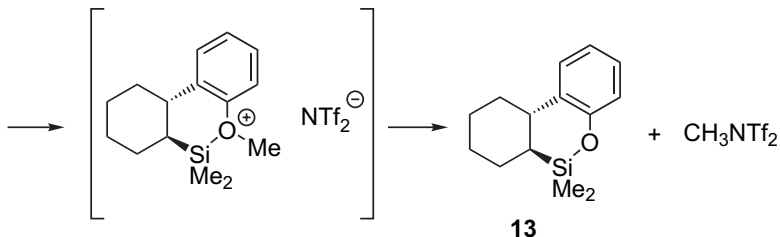

Scheme 9

The protodesilylation of (-)-3d did not give the expected triflimide 11d but a cyclic siloxane 13, which probably resulted from an intramolecular silylation of the methoxy substituent followed by an irreversible demethylation reaction (Scheme 9).

\section{Evaluation of the ENP silylated triflimides as catalysts for the Diels-Alder reaction}

We selected the cycloaddition of cyclopentadiene to methyl acrylate as model reaction. This should allow an easy comparison with the studies of Hawkins' group on related boron catalysts. $^{12}$

The catalysts $(10 \mathrm{~mol} \%)$ were formed in the solvent $\left(\mathrm{CH}_{2} \mathrm{Cl}_{2}\right.$ in most cases) of the Diels-Alder reaction (Scheme 10). Then $20 \mathrm{~mol} \%$ 2,6-di-tert-butyl-4-methylpyridine (DTBMP) was added to neutralise any residual Brönsted HNTf $_{2}$. Methyl acrylate ( 1 equiv) and then cyclopentadiene (4 equiv) were added at $-78^{\circ} \mathrm{C}$ (or $-100{ }^{\circ} \mathrm{C}$ ) and the mixture was left at that temperature. The results are summarised in Table 5.

Table 5. ENP silylated triflimides as catalysts for the cycloaddition of methyl acrylate and cyclopentadiene

\begin{tabular}{|c|c|c|c|c|c|c|}
\hline SM & Catalyst $\mathrm{X}=\mathrm{NTf}_{2}$ & Conditions & Yield, \% & endolexo & ee, $\%$ & $\begin{array}{l}\text { Product } \\
\text { configuration }\end{array}$ \\
\hline$(-)-7 \mathbf{a}$ & & $-78^{\circ} \mathrm{C}, \mathrm{CH}_{2} \mathrm{Cl}_{2}$ & 95 & 32 & 47 & $2 R$ \\
\hline$(-)-7 \mathbf{b}$ & & $\begin{array}{l}-78^{\circ} \mathrm{C}, \mathrm{CH}_{2} \mathrm{Cl}_{2} \\
-78^{\circ} \mathrm{C} \text {, toluene } \\
-10{ }^{\circ} \mathrm{C}, \mathrm{CH}_{2} \mathrm{Cl}_{2}\end{array}$ & $\begin{array}{l}96 \\
95 \\
94\end{array}$ & $\begin{array}{l}>100 \\
>100 \\
>100\end{array}$ & $\begin{array}{l}50 \\
44 \\
59\end{array}$ & $\begin{array}{l}2 R \\
2 R \\
2 R\end{array}$ \\
\hline$(-)-7 e$ & & $-78^{\circ} \mathrm{C}, \mathrm{CH}_{2} \mathrm{Cl}_{2}$ & 80 & 19 & 35 & $2 S$ \\
\hline$(+)-7 \mathbf{f}$ & & $-78^{\circ} \mathrm{C}, \mathrm{CH}_{2} \mathrm{Cl}_{2}$ & 95 & 49 & 40 & $2 S$ \\
\hline$(+)-7 g$ & & $-78^{\circ} \mathrm{C}, \mathrm{CH}_{2} \mathrm{Cl}_{2}$ & 95 & 99 & 49 & $2 S$ \\
\hline$(-)-7 g$ & $\mathrm{SiMe}_{2} \mathrm{X}$ & $-100{ }^{\circ} \mathrm{C}, \mathrm{CH}_{2} \mathrm{Cl}_{2}$ & 95 & 32 & 56 & $2 R$ \\
\hline$(-)-7 \mathbf{h}$ & & $-78^{\circ} \mathrm{C}$, toluene & 99 & 99 & 36 & $2 R$ \\
\hline$(-)-8 \mathbf{a}$ & $e_{2} X$ & $-78^{\circ} \mathrm{C}, \mathrm{CH}_{2} \mathrm{Cl}_{2}$ & 95 & 99 & 0 & - \\
\hline$(-)-8 b$ & $\mathrm{iMe}_{2} \mathrm{X}$ & $-78^{\circ} \mathrm{C}, \mathrm{CH}_{2} \mathrm{Cl}_{2}$ & 94 & 16 & 3 & - \\
\hline$(-)-8 c$ & $\mathrm{SiMe}_{2} \mathrm{X}$ & $-78^{\circ} \mathrm{C}, \mathrm{CH}_{2} \mathrm{Cl}_{2}$ & 95 & 49 & 7 & $2 S$ \\
\hline$(-)-9 \mathbf{a}$ & & $-78^{\circ} \mathrm{C}, \mathrm{CH}_{2} \mathrm{Cl}_{2}$ & 80 & 7.4 & 42 & $2 S$ \\
\hline$(+)-9 b$ & & $-78^{\circ} \mathrm{C}, \mathrm{CH}_{2} \mathrm{Cl}_{2}$ & 90 & 49 & 45 & $2 S$ \\
\hline
\end{tabular}




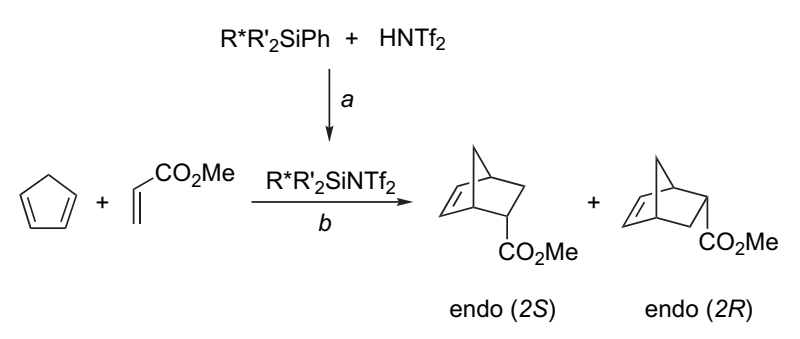

Scheme 10. Reagents and conditions: (a) 0.1 equiv of triflimides catalyst generated in situ at $\mathrm{rt}$ or $70-80^{\circ} \mathrm{C}$; (b) addition of methyl acrylate (1 equiv), cyclopentadiene (4 equiv), DTMB ( 0.2 equiv), $-78^{\circ} \mathrm{C}$ or $-100^{\circ} \mathrm{C}$.

The first conclusions of these experiments were that these chiral silyl triflimides were extremely active catalysts for the Diels-Alder reaction. In most cases, reactions were almost quantitative in less than $2 \mathrm{~h}$. This had already been demonstrated for the structurally more simple TMS and TIPSNTf ${ }_{2}$, which have been shown to be much better catalysts than the corresponding triflates. This can be assigned to a relief of I-strain in going from the trialkylsilyltriflimide to the corresponding silylated carbonyl complex (Scheme 11).

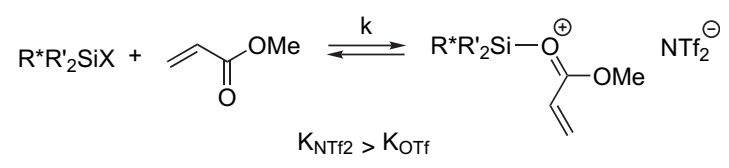

Scheme 11 .

All reactions were highly endo-selective. Enantiomeric excesses were measured by GC (CHIRASIL-DEX CB column) for the endo isomers only. The best enantiomeric excesses was $59 \%$, which is the highest enantioselectivity ever observed for a Diels-Alder reaction catalysed by a silicon Lewis acid. The selectivity was higher for catalysts having a 1-naphthyl group directly attached to the cycloalkane ring. Insertion of a methylene group allowing for more conformational mobility resulted in a significant decrease of facial selectivity. cis-Catalysts gave lower ee than the corresponding trans-catalysts. The presence of a phenoxy group on the phenyl substituent did not raise the selectivity in spite of a possible chelation of the oxygen with silicon.

An interesting observation was that catalyst derived from $(-)-7 \mathbf{a}$ and (-)-7b led to the same enantiomer although they have opposite configurations. This suggested different transition state structures in going from the phenyl to the naphthyl-substituted catalysts. The selectivity observed with the 1-naphthyl-substituted catalyst can be rationalised on the basis of a an approach of the reactants similar to that of the corresponding boron-catalysed reaction (Scheme $12):^{12 a}$ (1) both naphthalene and silyl groups are equatorial, (2) silicon complexes the carbonyl group anti to the $\mathrm{C}-\mathrm{O}$ ester bond, (3) the configuration of the enone unit is s-trans. ${ }^{27}$ This approach is favoured by electrostatic and dipole-induced attractions between the complexed carbonyl group and the polarisable naphthalene substituent. Thus the naphthalene substituent blocks the $\alpha$-face of the dienophile.

These attractive forces should be weaker when naphthyl $(17.6 \mathrm{D})$ is replaced by the less polarisable phenyl group (10.4 D). ${ }^{12 \mathrm{~b}}$ Lower energy conformations of the methyl methacrylate/(-)-11a complex were calculated to be $0.477 \mathrm{kcal} / \mathrm{mol}$ apart (Fig. 5). ${ }^{28}$

In the most stable conformation $\mathbf{A}$ (Fig. 5), the silicon atom coordinates to the carbonyl group syn to the methoxy group of a $s$-cis enone. In this conformation, the reactive double

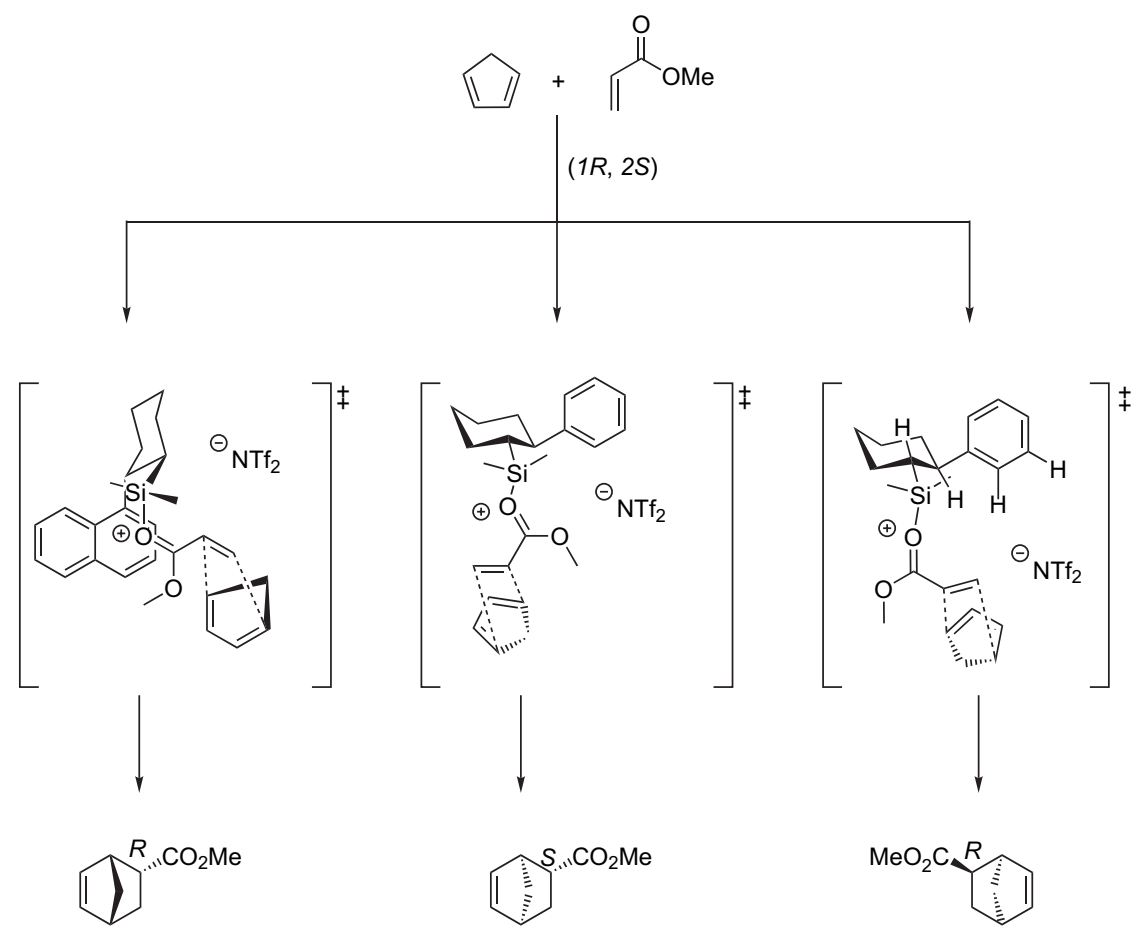

Scheme 12. Possible transition states for the catalysed cycloadditions. 


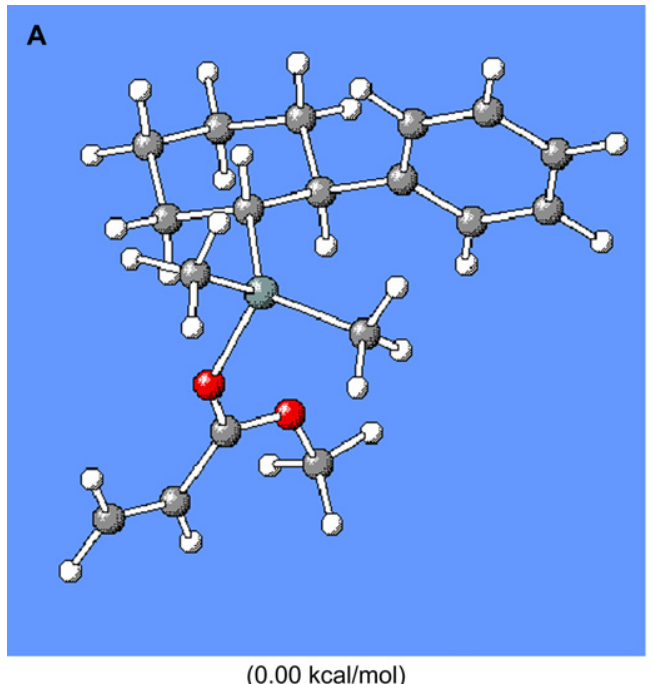

$(0.00 \mathrm{kcal} / \mathrm{mol})$

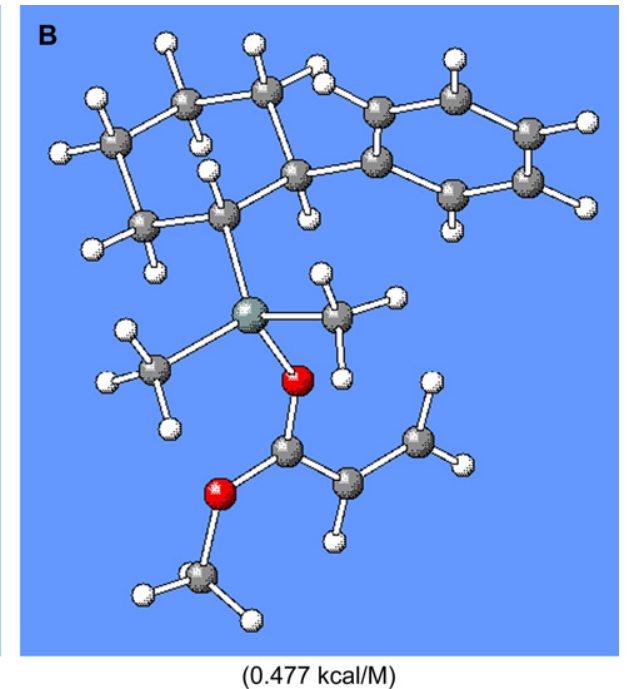

$(0.477 \mathrm{kcal} / \mathrm{M})$

Figure 5. Low energy conformations of methyl acrylate/(-)-11a complex.

bond is far away from the aryl group. Conformation $\mathbf{B}$ is only $0.477 \mathrm{kcal}$ higher than $\mathbf{A}$. It differs from $\mathbf{A}$ by the conformation around the olefin-ester bond, which brings the reactive double bond closer to the aryl group. Therefore we have to consider two additional transition states TS-2 and TS-3 (Scheme 12) leading to adducts of opposite configurations. We are conscious that this analysis is still pretty rough but it should help the design of new catalysts of this class, which should have structural features favouring one TS over the others. The replacement of the cyclohexane ring by a decaline would be an interesting possibility.

\section{Experimental}

\subsection{General}

All reactions requiring anhydrous or inert conditions were carried out under an atmosphere of dried argon in flamedried glassware. All solvents were dried by standard procedure and freshly distilled. Infrared spectra were recorded on a BIORAD FTS 135 FTIR. ${ }^{1} \mathrm{H}$ NMR spectra were recorded in $\mathrm{CDCl}_{3}$ on Varian Gemini-200, 300 or on Bruker AM-500 spectrometers at 200,300 or $500 \mathrm{MHz} .{ }^{13} \mathrm{C}$ NMR spectra were recorded at 50,75 or $125 \mathrm{MHz}$. Chemical shifts $(\delta)$ are given in parts per million relative to $\mathrm{Me}_{4} \mathrm{Si}\left(0,{ }^{1} \mathrm{H}\right)$ or $\mathrm{CDCl}_{3}\left(77.0,{ }^{13} \mathrm{C}\right)$. Mass spectra were recorded on Varian MAT-44 or Finnigan MAT-TSQ 70 spectrometer. Melting points were obtained on a Büchi Melting Point B-545 and were uncorrected. Thin-layer chromatography (TLC) was run on precoated silica gel plates $\left(\right.$ Merck $60 \mathrm{~F}_{254}$ ). Flash chromatography was carried out using flash silica gel 60 Merck $(40-63 \mu \mathrm{m})$. Chiral GC analyses were performed on CE instrument HRGC-5300 equipped with a flame ionisation detector and Merck Hitachi integrators using CHIRASIL-DEX CB column.

\subsection{Synthesis of 2-aryl-cycloalkenones 2}

General procedure: $23.03 \mathrm{~mL}(15.96 \mathrm{~g}, 57.59 \mathrm{mmol}) n-\mathrm{BuLi}$ in $2.5 \mathrm{~mol} / \mathrm{L}$ hexane was added at $0{ }^{\circ} \mathrm{C}$ to a solution of
$8.07 \mathrm{~mL}$ (5.83 g, $57.59 \mathrm{mmol}$ ) diisopropylamine in $140 \mathrm{~mL}$ dry THF under argon. The mixture was stirred for $30 \mathrm{~min}$ and then cooled to $-78^{\circ} \mathrm{C}$. Then, $5.87 \mathrm{~g}(52.35 \mathrm{mmol}) 2,3-$ epoxycyclohexanone was added slowly. The mixture was stirred for another $30 \mathrm{~min}$ at this temperature.

To a solution of $14.56 \mathrm{~mL}(21.68 \mathrm{~g}, 104.7 \mathrm{mmol})$ 1-bromonaphthalene in $140 \mathrm{~mL}$ THF in a three-neck $500 \mathrm{~mL}$ flask, $123.18 \mathrm{~mL}(1.7 \mathrm{M}, 81.30 \mathrm{~g}, 209.4 \mathrm{mmol}) t$-BuLi in pentane was added at $-78^{\circ} \mathrm{C}$ under argon. The reaction mixture was stirred for $1 \mathrm{~h}$ at $-78^{\circ} \mathrm{C}$, then $10 \mathrm{~min}$ at $0{ }^{\circ} \mathrm{C}$. The resulting mixture was then transformed to the above enolate solution through a cannula at $-78{ }^{\circ} \mathrm{C}$ under the pressure of argon. After this transformation was completed, the mixture was stirred at $-23{ }^{\circ} \mathrm{C}$ for $2 \mathrm{~h}$ and $50 \mathrm{~mL}$ saturated $\mathrm{NH}_{4} \mathrm{Cl}$ was added at $0{ }^{\circ} \mathrm{C}$. The mixture was extracted with $3 \times 100 \mathrm{~mL}$ $\mathrm{Et}_{2} \mathrm{O}$. The organic layers were washed with $2 \times 100 \mathrm{~mL}$ $3 \mathrm{~N} \mathrm{HCl}$, then $120 \mathrm{~mL}$ saturated $\mathrm{NaHCO}_{3}$ solution. The collected organic phases were dried, filtered and evaporated in vacuum.

The above resulting crude product was placed into a $500 \mathrm{~mL}$ flask with a Dean-Stark to separate the water. Toluene $(150 \mathrm{~mL})$ and $10 \mathrm{mg}$ para-toluenesulfonic acid (PTSA) were added. The mixture was heated under reflux for $2 \mathrm{~h}$. After cooling, $80 \mathrm{~mL}$ saturated $\mathrm{NaHCO}_{3}$ solution was added. The solution was extracted with $3 \times 100 \mathrm{~mL} \mathrm{Et}_{2} \mathrm{O}$ and the organic layer was dried and filtered. The solvent was removed under reduced pressure. The residue was chromatographed with $\mathrm{PE} / \mathrm{AcOEt}$ to give product $\mathbf{2 b}$.

5.2.1. 2-(1-Naphthyl)-cyclohexenone $2 \mathrm{~b}$. $\mathrm{RN}: 108842-56-$ 4; mp 107-108 ${ }^{\circ} \mathrm{C} ; 54 \%$. ${ }^{1} \mathrm{H} \mathrm{NMR}\left(\mathrm{CDCl}_{3}, 200 \mathrm{MHz}\right) \delta$ : 2.22 (pent, $2 \mathrm{H}, 6.6 \mathrm{~Hz}$ ), 2.61 (td, $2 \mathrm{H}, J=6.0,4.5 \mathrm{~Hz}$ ), 2.69 $(\mathrm{t}, 2 \mathrm{H}, J=6.4 \mathrm{~Hz}), 7.05(\mathrm{t}, 1 \mathrm{H}, J=4.5 \mathrm{~Hz}), 7.23(\mathrm{dd}, 1 \mathrm{H}$, $J=6.4,1.6 \mathrm{~Hz}), 7.37-7.48(\mathrm{~m}, 3 \mathrm{H}), 7.57-7.63(\mathrm{~m}, 1 \mathrm{H})$, $7.79-7.88(\mathrm{~m}, 2 \mathrm{H}) ;{ }^{13} \mathrm{C} \mathrm{NMR}\left(\mathrm{CDCl}_{3}, 50 \mathrm{MHz}\right) \delta: 23.09$, $26.43,38.78,125.20,125.50,125.60,125.78,126.99$, $128.17,128.28,131.98,133.46,135.11,140.49,149.97$, 198.03; IR (KBr): 780, 800, 1154, 1352, 1508, 1673, 2825, 2866, 2947, 3011, $3057 \mathrm{~cm}^{-1}$; MS (EI, $\left.70 \mathrm{eV}\right) \mathrm{m} / \mathrm{z}$ (\%): 152 (24), 165 (100), 178 (18), 194 (38), 222.1 (92). 
5.2.2. 2-(9-Anthracyl)-cyclohexenone 2c. $\mathrm{Mp} 156^{\circ} \mathrm{C}$ (decomposition); $15 \% .{ }^{1} \mathrm{H} \mathrm{NMR}\left(\mathrm{CDCl}_{3}, 200 \mathrm{MHz}\right) \delta: 2.38$ (pent, $2 \mathrm{H}, J=6.8 \mathrm{~Hz}), 2.77(\mathrm{td}, 2 \mathrm{H}, J=6.8,4.2 \mathrm{~Hz}), 2.82(\mathrm{t}$, $2 \mathrm{H}, J=6.8 \mathrm{~Hz}), 7.08(\mathrm{t}, 1 \mathrm{H}, J=4.2 \mathrm{~Hz}), 7.42(\mathrm{~m}, 4 \mathrm{H}), 7.79$ $(\mathrm{m}, 2 \mathrm{H}), 8.00(\mathrm{~m}, 2 \mathrm{H}), 8.44(\mathrm{~s}, 1 \mathrm{H}) ;{ }^{13} \mathrm{C} \mathrm{NMR}\left(\mathrm{CDCl}_{3}\right.$, $50 \mathrm{MHz}) \delta: 23.36,26.74,38.92,124.99$ (2C), 125.54 (2C), 125.71 (2C), 127.13, 128.66 (2C), 130.24 (2C), 131.37 (2C), 131.46, 138.56, 152.02, 198.12; IR (KBr): 735, 859, 1155, 1364, 1597, 1675, 2861, 2949, $3052 \mathrm{~cm}^{-1}$; MS (CI) $\mathrm{m} / \mathrm{z}$ (\%): 272.1 (24), 273.1 (100).

5.2.3. 2-(2-Phenoxy-phenyl)-cyclohexenone 2e. Mp 139$140{ }^{\circ} \mathrm{C} ; 31 \% .{ }^{1} \mathrm{H}$ NMR $\left(\mathrm{CDCl}_{3}, 200 \mathrm{MHz}\right) \delta: 2.07$ (pent, $2 \mathrm{H}, J=6.2 \mathrm{~Hz}), 2.41-2.54(\mathrm{~m}, 4 \mathrm{H}), 6.81(\mathrm{dd}, 1 \mathrm{H}, J=8.1$, $1.7 \mathrm{~Hz}), 6.89(\mathrm{t}, 1 \mathrm{H}, J=4.2 \mathrm{~Hz}), 6.99-7.36(\mathrm{~m}, 7 \mathrm{H}), 7.86$ $(\mathrm{d}, 1 \mathrm{H}, J=8.3 \mathrm{~Hz}) ;{ }^{13} \mathrm{C} \mathrm{NMR}\left(\mathrm{CDCl}_{3}, 50 \mathrm{MHz}\right) \delta: 22.77$, 26.15, 38.41, 117.83 (2C), 122.65 (2C), 128.43, 128.79 (2C), 130.98 (2C), 137.66, 148.44 (2C), 154.17 (2C), 197.18; IR (KBr): 754, 882, 1231, 1355, 1445, 1482, 1596, 1682, 2867, 2942, 3026, $3057 \mathrm{~cm}^{-1}$; MS (CI) $\mathrm{m} / \mathrm{z}$ (\%): 153.9 (100), 171.1 (13), 237.0 (5), 265.9 (3).

5.2.4. 4,4-Dimethyl-2-(1-naphthyl)-cyclohexenone $2 \mathrm{f}$. $\mathrm{Mp}$ 98-100 ${ }^{\circ} \mathrm{C} ; 46 \% .{ }^{1} \mathrm{H} \mathrm{NMR}\left(\mathrm{CDCl}_{3}, 200 \mathrm{MHz}\right) \delta: 1.32(\mathrm{~s}$, $6 \mathrm{H}, 2 \mathrm{Me}), 2.09(\mathrm{t}, 2 \mathrm{H}, J=6.6 \mathrm{~Hz}), 2.73(\mathrm{t}, 2 \mathrm{H}, J=6.6 \mathrm{~Hz})$, $6.74(\mathrm{~s}, 1 \mathrm{H}), 7.23(\mathrm{dd}, 1 \mathrm{H}, J=7.0,1.4 \mathrm{~Hz}), 7.41-7.49(\mathrm{~m}$, $3 \mathrm{H}), 7.57-7.62(\mathrm{~m}, 1 \mathrm{H}), 7.80-7.87(\mathrm{~m}, 2 \mathrm{H}) ;{ }^{13} \mathrm{C} \mathrm{NMR}$ $\left(\mathrm{CDCl}_{3}, 50 \mathrm{MHz}\right) \delta: 27.98$ (2C), 33.47, 35.02, 36.33, $125.11,125.34,125.53,125.77,127.06,128.12,128.29$, 132.09, 133.54, 134.84, 137.36, 159.03, 197.55; IR (KBr): $788,805,1146,1353,1466,1508,1592,1677,2863$, 2956, 3001, $3056 \mathrm{~cm}^{-1}$; MS (CI) $\mathrm{m} / \mathrm{z}(\%)$ : 94.9 (28), 140.9 (100), $251.0(6)$.

\subsection{Synthesis of trans-2-aryl-3-dialkylphenylsilyl cyclo- alkanones 3a-h}

General procedure (method $\boldsymbol{B}$ ): $50 \mathrm{~mL}$ solution of $\mathrm{PhMe}_{2^{-}}$ $\mathrm{SiLi}$ or $\mathrm{PhEt}_{2} \mathrm{SiLi}$ prepared from $6.4 \mathrm{~mL}$ (36.46 mmol) chlorodimethylphenylsilane or $\mathrm{PhEt}_{2} \mathrm{SiCl}$ and $0.63 \mathrm{~g}$ (91.15 mmol) lithium was added at $-30^{\circ} \mathrm{C}$ to a suspension of $1.63 \mathrm{~g}$ (18.23 mmol) CuCN (pre-dried) in $120 \mathrm{~mL} \mathrm{THF} \mathrm{in}$ a $500 \mathrm{~mL}$ three-neck flask under argon. The mixture was stirred for $1 \mathrm{~h}$ at $0{ }^{\circ} \mathrm{C}$ and cooled to $-40{ }^{\circ} \mathrm{C}$.

To the above mixture, was added slowly $10 \mathrm{~mL}$ solution of (1.57 g, $9.12 \mathrm{mmol}) \mathbf{2 a}$ in THF through a syringe. The reaction mixture was stirred for $2.5 \mathrm{~h}$ at $-23{ }^{\circ} \mathrm{C}$ and then for $2 \mathrm{~h}$ at $0{ }^{\circ} \mathrm{C}$. The reaction mixture was warmed to room temperature in $1 \mathrm{~h}$ and $60 \mathrm{~mL} \mathrm{MeOH}$ was added and the mixture was stirred for $15 \mathrm{~min}$. Then, $70 \mathrm{~mL} \mathrm{H}_{2} \mathrm{O}$ was added. The mixture was filtered through $5-6 \mathrm{~cm}$ Celite and washed with $\mathrm{CH}_{2} \mathrm{Cl}_{2}$. The filtrate was extracted with $3 \times 100 \mathrm{~mL}$ $\mathrm{CH}_{2} \mathrm{Cl}_{2}$. The combined organic layers were washed with brine, water, and dried over $\mathrm{MgSO}_{4}$, and concentrated under the reduced pressure. The residue was chromatographed with $\mathrm{PE} / \mathrm{Et}_{2} \mathrm{O}$ to give $1.99 \mathrm{~g}$ trans-3a (71\%).

5.3.1. trans-3-(Dimethylphenylsilyl)-2-phenyl-cyclohexanone 3a. Viscous oil; 71\%. ${ }^{1} \mathrm{H}$ NMR $\left(\mathrm{CDCl}_{3}, 500 \mathrm{MHz}\right)$ $\delta:-0.132$ (s, 3H, Me), -0.003 (s, 3H, Me), $1.70(\mathrm{~m}, 1 \mathrm{H})$, $1.77(\mathrm{td}, 1 \mathrm{H}, J=12.6,3.2 \mathrm{~Hz}), 1.86(\mathrm{~m}, 1 \mathrm{H}), 1.91(\mathrm{~m}, 1 \mathrm{H})$, $2.21(\mathrm{~m}, 1 \mathrm{H}), 2.43(\mathrm{td}, J=13.1,15.1,5.6 \mathrm{~Hz}), 2.54(\mathrm{dt}, 1 \mathrm{H}$,
$J=15.1,3.2,3.6 \mathrm{~Hz}), 3.42(\mathrm{~d}, 1 \mathrm{H}, 12.6 \mathrm{~Hz}), 6.99-7.05(\mathrm{~m}$, 2H), 7.22-7.41 (m, 8H); ${ }^{13} \mathrm{C} \mathrm{NMR}\left(\mathrm{CDCl}_{3}, 125 \mathrm{MHz}\right) \delta$ : $5.08,-3.10,27.36,29.92,34.47,42.12,58.08,127.01$, 127.52 (2C), 127.96 (2C), 128.81, 129.67 (2C), 133.82 (2C), 137.42, 137.99, 210.84; IR (film): 814, 837, 1110 , $1249,1427,1596,1710,2850,2925,3028,3066 \mathrm{~cm}^{-1}$; MS (EI, $70 \mathrm{eV)} \mathrm{m/z} \mathrm{( \% ):} 137.0$ (100), 211.0 (54), 308.1 (3); HRMS (CI) calculated for $\mathrm{C}_{20} \mathrm{H}_{24} \mathrm{OSi}$ : 308.1596, found: 308.1598. HPLC (Chiral AD column, hexane 98\%/i-PrOH $2 \%, 0.5 \mathrm{~mL} / \mathrm{min}): 5.86,7.47 \mathrm{~min} .(2 R, 3 R)-3 \mathrm{a}:[\alpha]_{\mathrm{D}}^{20}-87.8$ $(c 0.41, \mathrm{EtOH}),(2 S, 3 S)-3 \mathrm{a}:[\alpha]_{\mathrm{D}}^{20}+85(c 0.4, \mathrm{EtOH})$.

5.3.2. trans-3-(Dimethylphenylsilyl)-2-(1-naphthyl)cyclohexanone 3b. (Method A) viscous oil; 34\% (work-up with saturated $\mathrm{NH}_{4} \mathrm{Cl}$ solution at $\left.0{ }^{\circ} \mathrm{C}\right) .{ }^{1} \mathrm{H} \mathrm{NMR}\left(\mathrm{CDCl}_{3}\right.$, $500 \mathrm{MHz}) \delta:-026(\mathrm{~s}, 3 \mathrm{H}, \mathrm{Me}),-0.14(\mathrm{~s}, 3 \mathrm{H}, \mathrm{Me}), 1.77$ (m, 1H), $1.94(\mathrm{~m}, 1 \mathrm{H}, J=12.8,3.6 \mathrm{~Hz}), 2.01(\mathrm{~m}, 1 \mathrm{H}), 2.14$ $(\mathrm{td}, 1 \mathrm{H}, J=12.5,3.4 \mathrm{~Hz}), 2.24(\mathrm{~m}, 1 \mathrm{H}), 2.5(\mathrm{ddd}, 1 \mathrm{H}$, $J=14, \quad 12.8, \quad 5.8 \mathrm{~Hz}), 2.62(\mathrm{~m}, 1 \mathrm{H}), 4.14 \quad(\mathrm{~d}, 1 \mathrm{H}$, $J=12.5 \mathrm{~Hz}$ ), 7.18-7.45 (m, 9H), 7.68-7.75 (m, 2H), 7.79$7.85(\mathrm{~m}, 1 \mathrm{H}) ;{ }^{13} \mathrm{C} \mathrm{NMR}\left(\mathrm{CDCl}_{3}, 125 \mathrm{MHz}\right) \delta:-4.63$, $-3.28,27.82,29.53,33.64,42.29,55.3,124.02,125.03$, $125.13,125.55,127.54$ (2C), 127.95, 128.11, 128.83, 129.01, 131.97, 133.81 (2C), 134.09, 134.79, 137.39, 210.42; IR (film): 832, 1112, 1249, 1427, 1598 (C=C), 1709, 2856, 2931, 3046, $3067 \mathrm{~cm}^{-1}$; MS (EI, $\left.70 \mathrm{eV}\right) \mathrm{m} / \mathrm{z}$ (\%): 135.1 (10), 177.7 (10), 205.9 (56), 252 (40), 280 (56), 358.1 (100); HRMS (EI, $70 \mathrm{eV}$ ) calculated for $\mathrm{C}_{24} \mathrm{H}_{26} \mathrm{OSi}$ : 358.1752, found: 358.1763 . HPLC (Chiral AD column, hexane $90 \% / i$-PrOH 10\%, $1 \mathrm{~mL} / \mathrm{min}): 5.71,6.81 \mathrm{~min} .(2 R, 3 R)-$ 3b: $[\alpha]_{\mathrm{D}}^{20}-231$ (c 0.2 , toluene $),(2 S, 3 S)-3 \mathbf{b}:[\alpha]_{\mathrm{D}}^{20}+209$ (c 0.2 , toluene).

5.3.3. trans-2-(9-Anthracyl)-3-(dimethylphenylsilyl)cyclohexanone 3c. $\mathrm{Mp} 110-112{ }^{\circ} \mathrm{C} ; 20 \%$. ${ }^{1} \mathrm{H}$ NMR $\left(\mathrm{CDCl}_{3}, 500 \mathrm{MHz}\right) \delta:-0.65(\mathrm{~s}, 3 \mathrm{H}, \mathrm{Me}),-0.32(\mathrm{~s}, 3 \mathrm{H}$, $\mathrm{Me}), 1.90(\mathrm{~m}, 1 \mathrm{H}), 2.12(\mathrm{~m}, 1 \mathrm{H}), 2.18(\mathrm{~m}, 1 \mathrm{H}, J=13.2$, $3.4 \mathrm{~Hz}), 2.36(\mathrm{~m}, 3 \mathrm{H}), 2.61$ (ddd, $1 \mathrm{H}, J=12.7,13.2$, $3.0 \mathrm{~Hz}), 2.71(\mathrm{ddd}, 1 \mathrm{H}, J=13.2,6.3,15.3 \mathrm{~Hz}), 2.88(\mathrm{~m}$, $1 \mathrm{H}), 4.90(\mathrm{~d}, J=12.7 \mathrm{~Hz}), 6.86(\mathrm{dd}, 2 \mathrm{H}, J=7.9,1.3 \mathrm{~Hz})$, $7.06(\mathrm{t}, 2 \mathrm{H}, J=7.4 \mathrm{~Hz}), 7.19(\mathrm{~m}, 1 \mathrm{H}), 7.43(\mathrm{~m}, 4 \mathrm{H}), 7.85$ $(\mathrm{m}, 2 \mathrm{H}), 7.97(\mathrm{~m}, 2 \mathrm{H}), 8.04(\mathrm{~m}, 2 \mathrm{H}), 8.30(\mathrm{~s}, 1 \mathrm{H}) ;{ }^{13} \mathrm{C}$ NMR $\left(\mathrm{CDCl}_{3}, 125 \mathrm{MHz}\right) \delta:-5.11,-4.44,27.75,28.01$, 32.71, 41.97, 52.48, 123.53, 124.17 (2C), 124.39, 125.52, 126.92, 127.08 (2C), 127.67, 128.29, 129.29 (2C), 129.60, $130.46,131.50,131.61,131.80,133.02$ (2C), 136.98, 209.66; IR (KBr): 730, 816, 835, 1112, 1158, 1249, 1426, 1446, 1558, 1612, 1704, 2852, 2923, 3048, $3067 \mathrm{~cm}^{-1}$; MS (CI) m/z (\%): 58.9 (74), 98.9 (100), 136.8 (14), 209.0 (61), 231.0 (31), 393.3 (4), 408.3 (15), 409.4 (9); HRMS (CI) calculated for $\mathrm{C}_{28} \mathrm{H}_{28} \mathrm{OSi}$ : 408.1909, found: 408.1904 . HPLC (Chiral AD column, hexane 98\%/i-PrOH 2\%, $1 \mathrm{~mL} /$ $\min ): 16.78,28.58 \mathrm{~min}$. $(2 R, 3 R)-3 \mathrm{c}:[\alpha]_{\mathrm{D}}^{20}-140(c \quad 0.5$, $\left.\mathrm{CHCl}_{3}\right),(2 S, 3 S)-3 \mathrm{c}:[\alpha]_{\mathrm{D}}^{20}+142.6\left(c 0.54, \mathrm{CHCl}_{3}\right)$.

5.3.4. trans-2-(2-Methoxyphenyl)-3-(dimethylphenylsilyl)-cyclohexanone 3d. Viscous oil; 67\%. ${ }^{1} \mathrm{H}$ NMR $\left(\mathrm{CDCl}_{3}, 500 \mathrm{MHz}\right) \delta:-0.11$ (s, 3H, Me), 0.08 (s, 3H, Me), $1.68(\mathrm{qd}, 1 \mathrm{H}, J=12.7,3.5 \mathrm{~Hz}), 1.85(\mathrm{~m}, 1 \mathrm{H}), 1.90(\mathrm{~m}, 1 \mathrm{H})$, $1.98(\mathrm{td}, 1 \mathrm{H}, J=12.9,3.0 \mathrm{~Hz}), 2.18(\mathrm{~m}, 1 \mathrm{H}), 2.44(\mathrm{~m}, 1 \mathrm{H}$, $J=14.7,12.7,6.0 \mathrm{~Hz}), 2.57(\mathrm{~m}, 1 \mathrm{H}), 3.76(\mathrm{~s}, 3 \mathrm{H}, \mathrm{MeO})$, $3.82(\mathrm{~d}, 1 \mathrm{H}, J=12.9 \mathrm{~Hz}), 6.82(\mathrm{~d}, 1 \mathrm{H}, J=8.2 \mathrm{~Hz}), 6.90(\mathrm{t}$, $1 \mathrm{H}, J=7.4 \mathrm{~Hz}), 7.00(\mathrm{dd}, 1 \mathrm{H}, J=7.4,1.5 \mathrm{~Hz}), 7.22(\mathrm{td}, 1 \mathrm{H}$, 
$J=8.2,7.4,1.3 \mathrm{~Hz}), 7.30-7.40(\mathrm{~m}, 5 \mathrm{H}) ;{ }^{13} \mathrm{C} \mathrm{NMR}\left(\mathrm{CDCl}_{3}\right.$, $125 \mathrm{MHz}) \delta$ : $-5.08,-0.11,27.40,29.05,32.07,41.93$, $52.70,54.91,110.45,120.07,126.97,127.31$ (2C), 128.04, 128.50, 130.99, 133.65 (2C), 137.91, 156.93, 210.21; IR (film): 774, 827, 830, 1118, 1247, 1427, 1494, 1588, 1602, 1708, 2903, 2955, 3048, $3068 \mathrm{~cm}^{-1}$; MS (CI) m/z (\%): 58.9 (100), 135.1 (16), 261.0 (13), 323.1 (16), 338.1 (9), 339.2 (7); HRMS (CI) calculated for $\mathrm{C}_{21} \mathrm{H}_{26} \mathrm{O}_{2} \mathrm{Si}$ : 338.1702, found: 338.1697. HPLC (Chiral AD column, hexane $99 \% / i$-PrOH $1 \%, 1 \mathrm{~mL} / \mathrm{min})$ : $10.83,14.46 \mathrm{~min}$. $(2 R, 3 R)-3 d:[\alpha]_{\mathrm{D}}^{20}-84(c \quad 0.88, \mathrm{EtOH}),(2 S, 3 S)-3 d:[\alpha]_{\mathrm{D}}^{20}$ +74 (c 0.59, EtOH).

5.3.5. trans-2-(2-Phenoxyphenyl)-3-(dimethylphenylsilyl)-cyclohexanone 3e. Viscous oil; 57\%. ${ }^{1} \mathrm{H}$ NMR $\left(\mathrm{CDCl}_{3}, 500 \mathrm{MHz}\right) \delta:-0.05$ (s, 3H, Me), 0.21 (s, 3H, Me), $1.75(\mathrm{~m}, 1 \mathrm{H}, J=13.3,13.0 \mathrm{~Hz}), 1.85(\mathrm{~m}, 1 \mathrm{H}), 1.91(\mathrm{~m}$, $1 \mathrm{H}), 2.10(\mathrm{td}, 1 \mathrm{H}, J=13.0,2.9 \mathrm{~Hz}), 2.16(\mathrm{~m}, 1 \mathrm{H}), 2.38(\mathrm{td}$, $1 \mathrm{H}, \quad J=13.1, \quad 13.6,5.5 \mathrm{~Hz}), 2.49 \quad(\mathrm{ddd}, J=13.6, \quad 4.1$, $3.1 \mathrm{~Hz}), 3.90(\mathrm{~d}, 1 \mathrm{H}, J=13.0 \mathrm{~Hz}), 6.70(\mathrm{~d}, 1 \mathrm{H}, J=7.8 \mathrm{~Hz})$, $6.95(\mathrm{~m}, 1 \mathrm{H}), 7.04(\mathrm{~m}, 1 \mathrm{H}), 7.08(\mathrm{~m}, 2 \mathrm{H}), 7.13(\mathrm{~m}, 2 \mathrm{H})$, $7.15(\mathrm{~m}, 2 \mathrm{H}), 7.32(\mathrm{~m}, 3 \mathrm{H}), 7.39(\mathrm{~m}, 2 \mathrm{H}) ;{ }^{13} \mathrm{C}$ NMR $\left(\mathrm{CDCl}_{3}, 125 \mathrm{MHz}\right) \delta:-4.74,-2.80,27.70,29.22,31.71$, $41.92,53.60,117.3$ (2C), 118.47, 121.60, 122.71, 127.55 (2C), 128.18, 128.30 (2C), 128.69, 129.38, 131.60, 133.70 (2C), 138.25, 155.32, 156.10, 209.22; IR (film): 770, 813, $833,1112,1246,1427,1453,1488,1599,1710,2854$, 2925, 3046, $3075 \mathrm{~cm}^{-1}$; MS (CI) $\mathrm{m} / \mathrm{z}(\%)$ : 59.0 (100), 135.1 (8), 153.7 (28), 225 (19), 385.2 (5), 401.3 (5); HRMS (CI) calculated for $\mathrm{C}_{26} \mathrm{H}_{28} \mathrm{O}_{2} \mathrm{Si}: 400.1859$, found: 400.1918. HPLC (Chiral OD-H column, hexane 98\%/ $i$-PrOH 2\%, $1 \mathrm{~mL} / \mathrm{min})$ : 10.76, $13.66 \mathrm{~min} . \quad(2 R, 3 R)-3 \mathrm{e}:$ $\left.[\alpha]_{\mathrm{D}}^{20}-43(c) 2.0, \mathrm{EtOH}\right),(2 S, 3 S)-3 \mathrm{e}:[\alpha]_{\mathrm{D}}^{20}+45(c) 1.9$, $\mathrm{EtOH})$.

5.3.6. trans-3-(Dimethylphenylsilyl)-4,4-dimethyl-2-(1naphthyl)-cyclohexanone 3f. $\mathrm{Mp} 93-95{ }^{\circ} \mathrm{C}, 50 \% .{ }^{1} \mathrm{H}$ NMR $\left(\mathrm{CDCl}_{3}, 500 \mathrm{MHz}\right) \delta:-0.11(\mathrm{~s}, 3 \mathrm{H}, \mathrm{MeSi}), 0.30$ (s, $3 \mathrm{H}, \mathrm{MeSi}$ ), 1.12 (s, 3H, Me), 1.41 (s, 3H, Me), 1.86 (ddd, $J=13.6,3.8,6.4 \mathrm{~Hz}), 2.19(\mathrm{td}, J=13.6,11.7,6.2 \mathrm{~Hz}), 2.45$ (d, $J=11.1 \mathrm{~Hz}), 2.62$ (ddd, 1H, $J=16.0,3.8,6.2 \mathrm{~Hz}), 2.69$ (ddd, $1 \mathrm{H}, J=16.0,6.4,11.7 \mathrm{~Hz}), 4.50(\mathrm{~d}, 1 \mathrm{H}, J=11.1 \mathrm{~Hz})$, $7.12(\mathrm{~m}, 2 \mathrm{H}), 7.16(\mathrm{~m}, 2 \mathrm{H}), 7.18(\mathrm{~m}, 1 \mathrm{H}), 7.29(\mathrm{dd}, 1 \mathrm{H}$, $J=8.0,7.5 \mathrm{~Hz}), 7.36(\mathrm{~d}, 1 \mathrm{H}, J=7.0 \mathrm{~Hz}), 7.46(\mathrm{td}, 1 \mathrm{H}$, $J=7.5,1.0 \mathrm{~Hz}), 7.51(\mathrm{td}, 1 \mathrm{H}, J=6.9,1.3 \mathrm{~Hz}), 7.69(\mathrm{~d}$, $1 \mathrm{H}, J=8.1 \mathrm{~Hz}), 7.81(\mathrm{~d}, 1 \mathrm{H}, J=7.8 \mathrm{~Hz}), 7.96(\mathrm{~s}, 1 \mathrm{H}) ;{ }^{13} \mathrm{C}$ NMR $\left(\mathrm{CDCl}_{3}, 125 \mathrm{MHz}\right) \delta:-1.17,0.00,24.02,32.02$, 34.02, 36.60, 41.94, 43.24, 51.40, 123.99, 124.59, 124.92, $125.52,127.13$, (2C), 127.28, 127.83, 128.00, 128.75, $132.05,133.10$ (2C), 133.83, 134.83, 139.43, 211.27; IR (KBr): 775, 815, 831, 1110, 1250, 1426, 1511, 1597, 1708, 2864, 2952, 3044, $3066 \mathrm{~cm}^{-1}$; MS (CI) m/z (\%): 136.9 (69), 152.8 (26), 235.0 (100), 309.2 (40), 371.3 (16), 387.4 (10); HRMS (CI) calculated for $\mathrm{C}_{26} \mathrm{H}_{30} \mathrm{OSi}$ : 386.2066, found: 386.2070. HPLC (Chiral OD-H column, hexane 96\%/i-PrOH 4\%, $1 \mathrm{~mL} / \mathrm{min}): 14.36,17.82 \mathrm{~min}$. $(2 R, 3 S)-3 f$ : $[\alpha]_{\mathrm{D}}^{20}-63\left(c 0.87, \mathrm{CHCl}_{3}\right),(2 S, 3 R)-3 \mathrm{f}:[\alpha]_{\mathrm{D}}^{20}+61(c 0.85$, $\mathrm{CHCl}_{3}$ ).

5.3.7. trans-3-(Dimethylphenylsilyl)-2-(1'-naphthyl)cyclopentanone 3g. Viscous oil; 73\%. ${ }^{1} \mathrm{H} \mathrm{NMR}\left(\mathrm{CDCl}_{3}\right.$, $500 \mathrm{MHz}) \delta: 0.06$ (s, 3H, Me), 0.09 (s, 3H, Me), $1.89(\mathrm{~m}$, $1 \mathrm{H}), 2.18(\mathrm{~m}, 1 \mathrm{H}), 2.25(\mathrm{~m}, 1 \mathrm{H}), 2.51(\mathrm{~m}, 2 \mathrm{H}), 3.79(\mathrm{~d}$,
$1 \mathrm{H}, J=12.7 \mathrm{~Hz}), 7.23(\mathrm{~d}, 1 \mathrm{H}, J=7.0 \mathrm{~Hz}), 7.29(\mathrm{dd}, 2 \mathrm{H}$, $J=6.6,5.5 \mathrm{~Hz}), 7.35(\mathrm{~d}, 3 \mathrm{H}, J=7.0 \mathrm{~Hz}), 7.40(\mathrm{t}, 1 \mathrm{H}$, $J=6.6 \mathrm{~Hz}), 7.48(\mathrm{~m}, 2 \mathrm{H}), 7.76(\mathrm{~d}, 1 \mathrm{H}, J=8.0 \mathrm{~Hz}), 7.77(\mathrm{~m}$, $1 \mathrm{H}), 7.87(\mathrm{~m}, 1 \mathrm{H}) ;{ }^{13} \mathrm{C} \mathrm{NMR}\left(\mathrm{CDCl}_{3}, 125 \mathrm{MHz}\right) \delta:-5.03$, $-4.39,23.32,31.65,38.59,54.84,123.74,125.04,125.36$, $125.76,126.24,127.58$ (2C), 127.65, 128.89, 129.12, $131.89,133.89$ (2C), 134.05, 135.14, 136.14, 218.93; IR (film): 776, 838, 1250, 1427, 1596, 1737, 2852, 2924, 2956, 3046, $3050 \mathrm{~cm}^{-1}$; MS (CI) $\mathrm{m} / \mathrm{z}(\%): 58.7$ (100), 127.0 (16), 135.0 (14), 209.0 (75), 315.9 (3), 345.0 (3); HRMS (CI) calculated for $\mathrm{C}_{23} \mathrm{H}_{24} \mathrm{OSi}$ : 344.1596, found: 344.1596. HPLC (Chiral OD-H column, hexane 99\%/ $i$-PrOH 1\%, $1 \mathrm{~mL} / \mathrm{min}): 24.06,31.77 \mathrm{~min}$. (2R,3R)-3g: $[\alpha]_{\mathrm{D}}^{20}-9.1(c 0.55, \mathrm{EtOH}),(2 S, 3 S)-3 \mathrm{~g}:[\alpha]_{\mathrm{D}}^{20}+13.6(c 0.44$, $\mathrm{EtOH})$.

5.3.8. trans-3-(Diethylphenylsilyl)-2-phenyl-cyclohexanone 3h. $\mathrm{Mp} 44-46{ }^{\circ} \mathrm{C} ; 70 \%$. ${ }^{1} \mathrm{H} \mathrm{NMR}\left(\mathrm{CDCl}_{3}, 200 \mathrm{MHz}\right)$ $\delta$ : $0.095(\mathrm{~m}, 1 \mathrm{H}), 0.28(\mathrm{~m}, 1 \mathrm{H}), 0.64(\mathrm{~m}, 2 \mathrm{H}), 0.60-0.87$ (m, 6H), 1.71-2.04 (m, 4H), 2.18-2.52 (m, 3H), 3.31 (d, $1 \mathrm{H}, J=11.4 \mathrm{~Hz}), 6.93-6.98(\mathrm{dd}, 2 \mathrm{H}, J=7.6,5.7 \mathrm{~Hz}), 7.25-$ $7.36(\mathrm{~m}, 8 \mathrm{H}) ;{ }^{13} \mathrm{C} \mathrm{NMR}\left(\mathrm{CDCl}_{3}, 125 \mathrm{MHz}\right) \delta: 1.38,2.35$, $7.10,7.26,27.46,30.00,32.05,42.17,58.74,127.11$, 127.59 (2C), 127.88 (2C), 128.97, 129.76 (2C), 134.71 (2C), 134.94, 138.18, 211.07; IR (KBr): 716, 763, 1006, 1108, 1160, 1236, 1427, 1455, 1601, 1711, 2873, 2954, 3027, $3066 \mathrm{~cm}^{-1}$; MS (CI) $\mathrm{m} / \mathrm{z}(\%): 102.9$ (8), 156.9 (100), 162.9 (19), 259.1 (30), 307.2 (31), 336.1 (55), 337.2 (15); HRMS (CI) calculated for $\mathrm{C}_{22} \mathrm{H}_{28} \mathrm{OSi}$ : 336.1909, found: 336.1910. HPLC (Chiral AD column, hexane 99\%/ $i$-PrOH 1\%, $1 \mathrm{~mL} / \mathrm{min}): 12.14,14.97 \mathrm{~min} . \quad(2 R, 3 R)-3 \mathbf{h}:$ $[\alpha]_{\mathrm{D}}^{20}-73(c 1.0, \mathrm{EtOH}),(2 S, 3 S)-3 \mathbf{h}:[\alpha]_{\mathrm{D}}^{20}+69(c 1.0$, $\mathrm{EtOH})$.

5.3.9. cis-3-(Dimethylphenylsilyl)-2-phenyl-cyclohexanone 4a. (Method A: work-up with saturated $\mathrm{NH}_{4} \mathrm{Cl}$ solution at $0{ }^{\circ} \mathrm{C}$ ), mp $79-80{ }^{\circ} \mathrm{C}$ (racemic, but each enantiomer is viscous oil); 70\%. ${ }^{1} \mathrm{H} \mathrm{NMR}\left(\mathrm{CDCl}_{3}, 500 \mathrm{MHz}\right) \delta: 0.036(\mathrm{~s}, 3 \mathrm{H})$, $0.059(\mathrm{~s}, 3 \mathrm{H}), 1.75(\mathrm{ddd}, 1 \mathrm{H}, J=5.7,13.6,3.4 \mathrm{~Hz}), 1.81(\mathrm{dq}$, $1 \mathrm{H}, J=13.0,3.4,3.7,3.7 \mathrm{~Hz}), 1.85(\mathrm{~m}, 1 \mathrm{H}), 2.16(\mathrm{qd}, 1 \mathrm{H}$, $J=13.0,13.0,13.6,3.4 \mathrm{~Hz}), 2.31(\mathrm{~m}, 1 \mathrm{H}), 2.37(\mathrm{dt}, 1 \mathrm{H}$, $J=15.0,3.4,3.7 \mathrm{~Hz}), 2.77(\mathrm{td}, 1 \mathrm{H}, J=15.0,13.6,6.1 \mathrm{~Hz})$, $3.66(\mathrm{~d}, 1 \mathrm{H}, J=5.7 \mathrm{~Hz}), 7.18-7.38(\mathrm{~m}, 10 \mathrm{H}) ;{ }^{13} \mathrm{C} \mathrm{NMR}$ $\left(\mathrm{CDCl}_{3}, 125 \mathrm{MHz}\right) \delta:-4.15,-3.98,22.28,29.27,32.50$, 38.62, 58.17, 127.11, 127.58 (2C), 128.32 (2C), 128.89, 129.71 (2C), 133.66 (2C), 137.59, 138.26, 211.46; IR (KBr): 811, 833, 1112, 1248, 1427, 1598, 1706, 2862, 2951, 3024, $3067 \mathrm{~cm}^{-1}$; MS (+CI) m/z (\%): 74.9 (27), 134.9 (11), 148.9 (100), 211.0 (45), 293.0 (3), 308.1 (6, $\left.\mathrm{M}^{+}\right), 309.5\left(2, \mathrm{M}^{+}+1\right)$; Elemental analysis calculated for $\mathrm{C}_{20} \mathrm{H}_{24} \mathrm{OSi}$ : C $77.86 \%, \mathrm{H} 7.84 \%$; found: $\mathrm{C} 77.12 \%, \mathrm{H}$ 7.69\%. HPLC (chiral AD column, hexane 99.5\%/i-PrOH $0.5 \%, 1 \mathrm{~mL} / \mathrm{min}): 18.36,26.57 \mathrm{~min}$. $[\alpha]_{\mathrm{D}}^{20}+16.3$ (c 1.1 , $\mathrm{EtOH}),[\alpha]_{\mathrm{D}}^{20}-15.9(c 1.35, \mathrm{EtOH})$.

5.3.10. cis-3-(Dimethylphenylsilyl)-2-(1-naphthyl)-cyclohexanone 4b. (Method $\mathrm{A}$ ), viscous oil, 23\%. ${ }^{1} \mathrm{H}$ NMR $\left(\mathrm{CDCl}_{3}, 200 \mathrm{MHz}\right) \delta: 0.034(\mathrm{~s}, 3 \mathrm{H}), 0.17(\mathrm{~s}, 3 \mathrm{H}), 1.89-$ $2.17(\mathrm{~m}, 3 \mathrm{H}), 2.23-2.44(\mathrm{~m}, 3 \mathrm{H}), 2.78(\mathrm{td}, 1 \mathrm{H}, J=13.2$, $13.2,5.0 \mathrm{~Hz}), 4.57(\mathrm{~d}, 1 \mathrm{H}, J=5.6 \mathrm{~Hz}), 7.17-7.51(\mathrm{~m}, 8 \mathrm{H})$, 7.72-7.84 (m, 3H), 8.16-8.21 (m, 1H); ${ }^{13} \mathrm{C} \mathrm{NMR}\left(\mathrm{CDCl}_{3}\right.$, $50 \mathrm{MHz})$ : $-3.62,-3.18,24.09,30.53,33.78,38.06,52.03$, $124.21,124.62,125.59,126.49,126.99,127.55$ (2C), 
127.99, 128.62, 128.90, 132.50, 133.68 (2C), 133.88, 134.53, 137.75, 212.07; IR (film): 791, 832, 1118, 1254, 1427, 1593, 1708, 2854, 2925, 2954, 3048, $3068 \mathrm{~cm}^{-1}$; MS (+CI) m/z (\%): 135.0 (17), 207.1 (100), 273 (40), $358.2\left(30, \mathrm{M}^{+}\right), 359.1\left(10, \mathrm{M}^{+}+1\right)$; HRMS (CI) calculated for $\mathrm{C}_{24} \mathrm{H}_{26} \mathrm{OSi}$ : 358.1752, found: 358.1760. HPLC (chiral AS column, hexane 95\%/i-PrOH 5\%, $1 \mathrm{~mL} / \mathrm{min}): 6.77$, $14.16 \mathrm{~min} ;[\alpha]_{\mathrm{D}}^{20}-7.8$ (c 1.0, EtOH, Peak1), +8.2 (c 1.0, EtOH, Peak2).

\subsection{Synthesis of trans-2-arylmethyl-3-dialkylphenylsilyl cycloalkanones 5 a-c}

General procedure: under argon, to a suspension of $17.56 \mathrm{~g}$ (92.21 mmol, 1.5 equiv) $\mathrm{CuI}$ in $120 \mathrm{~mL}$ dry THF and $40 \mathrm{~mL}$ hexamethylphosphoramide (HMPA) in a $500 \mathrm{~mL}$ three-neck flask, was added at $-30{ }^{\circ} \mathrm{C}, 120 \mathrm{~mL}$ solution of dimethylphenylsilyllithium prepared from $31 \mathrm{~mL}(31.48 \mathrm{~g}$, $184.41 \mathrm{mmol}, 3$ equiv) chlorodimethylphenylsilane and $3.84 \mathrm{~g}$ (553.23 mmol, 9 equiv) lithium. The mixture was stirred for $1 \mathrm{~h}$ at $0{ }^{\circ} \mathrm{C}$ and cooled down to $-78^{\circ} \mathrm{C}$. Then $5.95 \mathrm{~mL}$ (5.91 g, $61.47 \mathrm{mmol}, 1$ equiv) 2-cyclohexene-1one was slowly added through a syringe. The reaction mixture was warmed to $-23^{\circ} \mathrm{C}$ and continued to stir for $3 \mathrm{~h}$ and cooled down to $-78^{\circ} \mathrm{C}$. Then, $21 \mathrm{~mL}(29.62 \mathrm{~g}$, $127.73 \mathrm{mmol}, 2.82$ equiv) benzyl bromide was added dropwise. After being stirred for $5 \mathrm{~h}$ at $-23{ }^{\circ} \mathrm{C}$ and $15 \mathrm{~min}$ at $0{ }^{\circ} \mathrm{C}$, the reaction was stopped with $100 \mathrm{~mL}$ saturated $\mathrm{NH}_{4} \mathrm{Cl}$ solution. The mixture was filtered through a pad of Celite and washed with $\mathrm{CH}_{2} \mathrm{Cl}_{2}$. The filtrate was extracted with $3 \times 150 \mathrm{~mL} \mathrm{CH}_{2} \mathrm{Cl}_{2}$, washed with brine and water. The collected extracts were dried, filtered and evaporated, the crude product was flash chromatographed to give $17.0 \mathrm{~g}$ 5a $\left(85 \%\right.$ yield, $\left.R_{f}=0.31, \mathrm{EP} / \mathrm{Et}_{2} \mathrm{O}=8 / 1\right)$ as a white solid.

5.4.1. trans-2-Benzyl-3-(dimethylphenylsilyl)-cyclohexanone 5a. Mp 55-58 ${ }^{\circ} \mathrm{C} ; 85 \%$. ${ }^{1} \mathrm{H} \mathrm{NMR}\left(\mathrm{CDCl}_{3}, 500 \mathrm{MHz}\right.$, $\left.42{ }^{\circ} \mathrm{C}\right) \delta: 0.349(\mathrm{~s}, 3 \mathrm{H}), 0.357(\mathrm{~s}, 3 \mathrm{H}), 1.42(\mathrm{td}, 1 \mathrm{H}, J=8.6$, 8.6, $3.8 \mathrm{~Hz}), 1.70(\mathrm{~m}, 1 \mathrm{H}), 1.84(\mathrm{~m}, 1 \mathrm{H}), 1.99(\mathrm{~m}, 2 \mathrm{H})$, $2.28(\mathrm{~m}, 1 \mathrm{H}), 2.41(\mathrm{~m}, 1 \mathrm{H}), 2.72(\mathrm{~m}, 2 \mathrm{H}), 2.90(\mathrm{dd}, 1 \mathrm{H}$, $J=4.4,14.8 \mathrm{~Hz}), 7.0(\mathrm{dd}, 2 \mathrm{H}, J=7.4,1.4 \mathrm{~Hz}), 7.14(\mathrm{~m}$, $1 \mathrm{H}), 7.20(\mathrm{~m}, 2 \mathrm{H}), 7.35(\mathrm{~m}, 3 \mathrm{H}), 7.45(\mathrm{dd}, 2 \mathrm{H}, J=7.9$, $1.5 \mathrm{~Hz}) ;{ }^{13} \mathrm{C} \mathrm{NMR}\left(\mathrm{CDCl}_{3}, 125 \mathrm{MHz}, 42^{\circ} \mathrm{C}\right) \delta:-3.28$, $-2.93,25.21,28.70,30.95,36.42,41.04,53.56,125.81$, 128.03 (2C), 127.85 (2C), 128.91 (2C), 129.03, 133.66 (2C), 137.81, 140.02, 213.38; IR (KBr): 734, 773, 812, 832 , 933, 1111, 1250, 1427, 1453, 1495, 1603, 1709, 2858, 2950, 3025, $3066 \mathrm{~cm}^{-1}$; MS (CI) m/z (\%): 135.0 (11), 171.0 (100), 245.1 (56), 307.1 (24), 322.1 (2), 323.2 (16); HRMS (CI) calculated for $\mathrm{C}_{21} \mathrm{H}_{26} \mathrm{OSi}$ : 322.1752, found: 322.1744. HPLC (Chiral OD-H column, hexane $99 \% / i-\mathrm{PrOH} 1 \%, 1 \mathrm{~mL} / \mathrm{min})$ : $12.69,15.41 \mathrm{~min} .(2 R, 3 R)-$ 5a: $[\alpha]_{\mathrm{D}}^{20}-79(c 1.0, \mathrm{EtOH}),(2 S, 3 S)-\mathbf{5 a}:[\alpha]_{\mathrm{D}}^{20}+86(c 1.0$, $\mathrm{EtOH})$.

5.4.2. trans-3-(Dimethylphenylsilyl)-2-(2-naphthylmethyl)-cyclohexanone 5b. Mp 92-93 ${ }^{\circ} \mathrm{C} ; 41 \% .{ }^{1} \mathrm{H}$ NMR $\left(\mathrm{CDCl}_{3}, 500 \mathrm{MHz}\right) \delta: 0.36(\mathrm{~s}, 3 \mathrm{H}), 0.37(\mathrm{~s}, 3 \mathrm{H}), 1.44(\mathrm{td}$, $1 \mathrm{H}, J=8.6,8.6,3.8 \mathrm{~Hz}), 1.73(\mathrm{~m}, 1 \mathrm{H}), 1.86(\mathrm{~m}, 1 \mathrm{H}), 2.02$ $(\mathrm{m}, 2 \mathrm{H}), 2.29(\mathrm{ddd}, 1 \mathrm{H}, J=14.4,9.7,5.3 \mathrm{~Hz}), 2.41(\mathrm{td}, 1 \mathrm{H}$, $J=6.2,6.2,14.4 \mathrm{~Hz}), 2.80(\mathrm{td}, 1 \mathrm{H}, J=8.6,8.4,5.4 \mathrm{~Hz})$, $2.84(\mathrm{dd}, 1 \mathrm{H}, J=5.4,13.6 \mathrm{~Hz}), 3.03(\mathrm{dd}, 1 \mathrm{H}, J=8.4$,
$13.6 \mathrm{~Hz}), 7.12(\mathrm{dd}, 1 \mathrm{H}, J=8.4,1.3 \mathrm{~Hz}), 7.32(\mathrm{t}, 2 \mathrm{H}$, $J=7.4 \mathrm{~Hz}), 7.36(\mathrm{~d}, 1 \mathrm{H}, J=1.3 \mathrm{~Hz}), 7.39(\mathrm{~m}, 1 \mathrm{H}), 7.41(\mathrm{~m}$, 1H), $7.43(\mathrm{~m}, 1 \mathrm{H}), 7.45(\mathrm{dd}, 2 \mathrm{H}, J=7.9,1.5 \mathrm{~Hz}), 7.66(\mathrm{~d}$, $1 \mathrm{H}, J=8.4 \mathrm{~Hz}), 7.69(\mathrm{~d}, 1 \mathrm{H}, J=7.6 \mathrm{~Hz}), 7.76(\mathrm{~d}, 1 \mathrm{H}$, $J=7.5 \mathrm{~Hz}) ;{ }^{13} \mathrm{C} \mathrm{NMR}\left(\mathrm{CDCl}_{3}, 125 \mathrm{MHz}\right) \delta:-3.18,-2.74$, $25.42,29.00,31.11,36.23,41.23,53.68,125.05,125.60$, 127.20, 127.39 (2C), 127.52, 127.55, 127.86 (2C), 129.07, $131.85,133.24,133.69$ (2C), 137.59, 137.68, 213.64; IR (KBr): 735, 782, 817, 1039, 1114, 1251, 1427, 1505, 1601, $1634,1693,2854,2910,2949,3023,3063 \mathrm{~cm}^{-1}$; MS (+CI) m/z (\%): 135.0 (27), 141.0 (100), 295.0 (24), 373.4 (22, $\left.\mathrm{M}^{+}+1\right)$; HRMS (CI) calculated for $\mathrm{C}_{25} \mathrm{H}_{28} \mathrm{OSi}$ : 372.1909, found: 372.1894. HPLC (OD-H column, hexane 99\%/i-PrOH 1\%, $1 \mathrm{~mL} / \mathrm{min}): 11.24,13.37 \mathrm{~min}$. $(2 R, 3 R)-$ 5b: $[\alpha]_{\mathrm{D}}^{20}-89(c 1.0, \mathrm{EtOH}),(2 S, 3 S)-5 \mathbf{b}:[\alpha]_{\mathrm{D}}^{20}+67(c$ $0.53, \mathrm{EtOH})$.

5.4.3. trans-3-(Dimethylphenylsilyl)-2-(1-naphthylmethyl)-cyclohexanone 5c. Viscous oil; 39\%. ${ }^{1} \mathrm{H}$ NMR $\left(\mathrm{CDCl}_{3}, 500 \mathrm{MHz}\right) \delta: 0.22(\mathrm{~s}, 3 \mathrm{H}), 0.26(\mathrm{~s}, 3 \mathrm{H}), 1.53(\mathrm{q}$, $1 \mathrm{H}, J=6.2 \mathrm{~Hz}), 1.79(\mathrm{~m}, 1 \mathrm{H}), 1.97(\mathrm{~m}, 2 \mathrm{H}), 2.14(\mathrm{~m}, 1 \mathrm{H})$, $2.35(\mathrm{dt}, 1 \mathrm{H}, J=14.1,6.0,6.0 \mathrm{~Hz}), 2.54(\mathrm{~m}, 1 \mathrm{H}), 3.00(\mathrm{q}$, $1 \mathrm{H}, J=7.8,7.4 \mathrm{~Hz}), 3.18(\mathrm{dd}, 1 \mathrm{H}, J=7.4,14.0 \mathrm{~Hz}), 3.40$ $(\mathrm{dd}, 1 \mathrm{H}, J=7.8,14.0 \mathrm{~Hz}), 7.17(\mathrm{~d}, 1 \mathrm{H}, J=7.0 \mathrm{~Hz}), 7.21(\mathrm{~m}$, 2H), $7.29(\mathrm{~m}, 3 \mathrm{H}), 7.34(\mathrm{dd}, 1 \mathrm{H}, J=7.0,8.2 \mathrm{~Hz}), 7.40(\mathrm{~m}$, $1 \mathrm{H}), 7.44(\mathrm{~m}, 1 \mathrm{H}), 7.70(\mathrm{~d}, 1 \mathrm{H}, J=8.2 \mathrm{~Hz}), 7.79(\mathrm{~d}, 1 \mathrm{H}$, $J=8.3 \mathrm{~Hz}), 7.82(\mathrm{dd}, 1 \mathrm{H}, \quad J=8.6,1.4 \mathrm{~Hz}) ;{ }^{13} \mathrm{C} \mathrm{NMR}$ $\left(\mathrm{CDCl}_{3}, 125 \mathrm{MHz}\right) \delta:-3.58,-3.15,23.72,27.48,29.78$, $34.66,40.12,51.56,123.57,124.95,125.27,125.76$, $126.57,126.94,127.68$ (2C), 128.57, 128.87, 131.80, 133.46 (2C), 133.86, 135.08, 137.53, 213.53; IR (film): 732, 777, 810, 832, 1110, 1250, 1426, 1596, 1653, 1706, 2856, 2949, 3030, $3064 \mathrm{~cm}^{-1}$; MS (+CI) m/z (\%): 95 (22), 134.9 (44), 140.9 (60), 221.0 (100), 357.1 (12), 372.6 (42), $373.1\left(44, \mathrm{M}^{+}+1\right)$; HRMS (CI) calculated for $\mathrm{C}_{25} \mathrm{H}_{28} \mathrm{OSi}$ : 372.1909, found: 372.1892. HPLC (OD-H column, Hexane 99\%/i-PrOH 1\%, $1 \mathrm{~mL} / \mathrm{min}): 16.15,18.02 \mathrm{~min} .(2 R, 3 R)-5 \mathbf{c}$ : $[\alpha]_{\mathrm{D}}^{20}-95.7(c 0.6, \mathrm{EtOH}),(2 S, 3 S)-5 \mathrm{c}:[\alpha]_{\mathrm{D}}^{20}+97(c 0.62$, $\mathrm{EtOH})$.

\subsection{Synthesis of ENP trans-1-dialkylphenylsilyl-2-aryl- cycloalkanes $7 \mathrm{a}-\mathrm{h}$ and trans-1-dialkylphenylsilyl-2- arylmethyl-cyclohexanones $8 \mathrm{a}-\mathrm{c}$}

General procedure: to a $50 \mathrm{~mL}$ one-neck flask with a stirring bar and a condenser, were added $0.787 \mathrm{~g}$ (2.194 mmol, 1 equiv) (+) (2S,3S)-3b, $0.505 \mathrm{~g} \quad(97 \%, 2.632 \mathrm{mmol}$, 1.2 equiv) para-toluenesulfonhydrazide in $15 \mathrm{~mL} \mathrm{EtOH}$. The solution was heated at $60^{\circ} \mathrm{C}$ for $2 \mathrm{~h}$ (controlled by TLC) and cooled to room temperature. The solvent was removed under the reduced pressure to afford the corresponding tosylhydrazone, which was used for the next step without isolation.

To the solution of the obtained tosylhydrazone in $20 \mathrm{~mL}$ $\mathrm{EtOH} / \mathrm{MeOH} \quad(1: 1), \quad 0.726 \mathrm{~g} \quad(10.97 \mathrm{mmol}, \quad 5$ equiv $)$ $\mathrm{NaBH}_{3}(\mathrm{CN}), 0.747 \mathrm{~g}$ (5.485 mmol, 2.5 equiv) $\mathrm{ZnCl}_{2}$ were added. The mixture was heated at $60{ }^{\circ} \mathrm{C}$ for $3 \mathrm{~h}$ and cooled to room temperature. The mixture was filtered through 5 $6 \mathrm{~cm}$ Celite and washed with $\mathrm{CH}_{2} \mathrm{Cl}_{2}$. After concentration, the residue was diluted with $5 \mathrm{~mL} \mathrm{CH}_{2} \mathrm{Cl}_{2}$ and $5 \mathrm{~mL}$ saturated $\mathrm{NaHCO}_{3}$ solution. The mixture was filtered through $5-6 \mathrm{~cm}$ Celite and washed with $\mathrm{CH}_{2} \mathrm{Cl}_{2}$. The filtrate was 
extracted with $3 \times 10 \mathrm{~mL} \mathrm{CH}_{2} \mathrm{Cl}_{2}$ and the collected organic layers were dried over $\mathrm{MgSO}_{4}$, filtered and evaporated. Flash chromatograph of the residue afforded $0.34 \mathrm{~g} \mathrm{(+)}$ ( $1 S, 2 R)-9 b$ ( $45 \%$, viscous oil, $R_{f}=0.57 /$ hexane).

\subsection{1. trans-1-(Dimethylphenylsilyl)-2-phenyl-cyclohex-} ane 7a. $(1 R, 2 S)-7 \mathbf{a}: 44 \%$, viscous oil, $[\alpha]_{\mathrm{D}}^{20}+33.3(c 0.21$, $\mathrm{EtOH}) ;(1 S, 2 R)-7 \mathbf{a}: 31 \%$, viscous oil, $[\alpha]_{\mathrm{D}}^{20}-38.6(c 0.44$, $\mathrm{EtOH})$.

${ }^{1} \mathrm{H}$ NMR $\left(\mathrm{CDCl}_{3}, 500 \mathrm{MHz}\right) \delta:-0.18(\mathrm{~s}, 3 \mathrm{H}), 0.004(\mathrm{~s}, 3 \mathrm{H})$, $1.28(\mathrm{~m}, 3 \mathrm{H}), 1.38(\mathrm{~m}, 1 \mathrm{H}), 1.48(\mathrm{~m}, 1 \mathrm{H}), 1.83(\mathrm{~m}, 4 \mathrm{H}), 2.46$ $(\mathrm{td}, 1 \mathrm{H}, J=8.2,8.2,11.0 \mathrm{~Hz}), 7.10-7.28(\mathrm{~m}, 5 \mathrm{H}), 7.30-7.45$ $(\mathrm{m}, 5 \mathrm{H}) ;{ }^{13} \mathrm{C} \mathrm{NMR}\left(\mathrm{CDCl}_{3}, 125 \mathrm{MHz}\right) \delta:-4.88,-2.96$, $26.96,27.43,28.31,31.11,37.94,46.69,125.98,127.27$ (2C), 127.77 (2C), 128.06 (2C), 128.28, 133.84 (2C), 139.02, 147.47; IR (film): 775, 813, 834, 1111, 1248, $1427,1445,1491,1600,2849,2922,3025,3066 \mathrm{~cm}^{-1}$; MS (+CI) $m / z(\%): 134.8$ (27), 136.1 (5), 216.9 (100), 279.0 (24), 294.2 (2), 295.6 (1); HRMS (CI) calculated for $\mathrm{C}_{20} \mathrm{H}_{26} \mathrm{Si}:$ 294.1803, found: 294.1794.

5.5.2. trans-1-(Dimethylphenylsilyl)-2-(1-naphthyl)-cyclohexanone $7 \mathbf{b} .(1 S, 2 R)-7 \mathbf{b}: 45 \%$, viscous oil, $[\alpha]_{\mathrm{D}}^{20}+35.7$ (c $0.7, \mathrm{EtOH}) ;(1 R, 2 S)-7 \mathbf{b}: 39 \%$, viscous oil, $[\alpha]_{\mathrm{D}}^{20}-32.8$ (c $0.7, \mathrm{EtOH})$.

${ }^{1} \mathrm{H} \mathrm{NMR}\left(\mathrm{CDCl}_{3}, 200 \mathrm{MHz}\right) \delta:-0.33(\mathrm{~s}, 3 \mathrm{H}), 0.13$ (s, 3H), 1.15-1.52 (m, 5H), 1.70-1.95 (m, 4H), 3.20-3.40 (br m, $1 \mathrm{H}), 7.02-7.16(\mathrm{~m}, 3 \mathrm{H}), 7.17-7.20(\mathrm{~m}, 2 \mathrm{H}), 7.22-7.32(\mathrm{~m}$, 2H), 7.40-7.50 (m, 2H), $7.64(\mathrm{~d}, 1 \mathrm{H}, J=7.4 \mathrm{~Hz}), 7.80-$ 7.88 (dd, $1 \mathrm{H}, J=5.8,3.8 \mathrm{~Hz}), 8.00-8.01(\mathrm{~m}, 1 \mathrm{H}) ;{ }^{13} \mathrm{C}$ NMR $\left(\mathrm{CDCl}_{3}, 50 \mathrm{MHz}\right) \delta:-4.23,-2.89,27.45,27.75$, 29.00, 30.82, 38.55, 39.39, 122.91, 124.84, 125.07, 125.37, $125.44,126.24,127.29$ (2C), 128.29, 128.89, 130.93, 133.78 (2C), 134.00, 139.05, 143.60; IR (film): 776, 817, $834,1112,1248,1427,1444,1511,1596,2848,2924$, 3046, $3067 \mathrm{~cm}^{-1}$; MS (EI, $\left.70 \mathrm{eV}\right) \mathrm{m} / \mathrm{z}(\%)$ : 83.8 (100), 135.1 (21), 208.1 (23), 267.2 (86), 271.1 (51), 329.1 (2), 344.2 (12).

5.5.3. trans -1-(Dimethylphenylsilyl)-2-(2-methoxyphenyl)-cyclohexane $7 d$. $(1 R, 2 S)-7 d$ : $57 \%$, viscous oil, $[\alpha]_{\mathrm{D}}^{20}+20(c 0.50, \mathrm{EtOH}) ;(1 S, 2 R)-7 \mathbf{d}: 51 \%$, viscous oil, $[\alpha]_{\mathrm{D}}^{20}-13.3(c$ 1.2, EtOH).

${ }^{1} \mathrm{H}$ NMR $\left(\mathrm{CDCl}_{3}, 500 \mathrm{MHz}\right) \delta:-0.195(\mathrm{~s}, 3 \mathrm{H}), 0.032(\mathrm{~s}$, $3 \mathrm{H}), 1.25(\mathrm{~m}, 2 \mathrm{H}), 1.38(\mathrm{~m}, 2 \mathrm{H}), 1.49(\mathrm{~m}, 1 \mathrm{H}), 1.76(\mathrm{~m}$, $4 \mathrm{H}), 3.01(\mathrm{td}, 1 \mathrm{H}, J=11.0,11.0,3.1 \mathrm{~Hz}), 3.77(\mathrm{~s}, 3 \mathrm{H}), 6.76$ $(\mathrm{d}, 1 \mathrm{H}, J=8.2 \mathrm{~Hz}), 6.82(\mathrm{td}, 1 \mathrm{H}, J=7.4,7.4,1.1 \mathrm{~Hz}), 7.09$ (td, $1 \mathrm{H}, J=7.4,7.4,1.7 \mathrm{~Hz}), 7.12(\mathrm{dd}, 1 \mathrm{H}, J=7.4,1.7 \mathrm{~Hz})$, $7.25(\mathrm{~m}, 3 \mathrm{H}), 7.32(\mathrm{~m}, 2 \mathrm{H}) ;{ }^{13} \mathrm{C} \mathrm{NMR}\left(\mathrm{CDCl}_{3}, 125 \mathrm{MHz}\right)$ $\delta:-4.78,-3.25,27.20,27.66,28.66,30.00,36.43,38.68$, $54.97,110.49,120.40,126.60,127.16$ (2C), 128.06, 128.78, 133.74, 133.75 (2C), 139.67, 156.57; IR (film): 769, 815, 834, 1111, 1243, 1427, 1439, 1463, 1491, 1585, 1599, 2847, 2925, 3033, $3067 \mathrm{~cm}^{-1}$; MS (+CI) m/z (\%): 120.68 (64), 134.8 (22), 136.8 (14), 188.9 (79), 247.0 (100), 309.1 (41), 324.1 (13), 325.1 (7); HRMS (CI) calculated for $\mathrm{C}_{21} \mathrm{H}_{28} \mathrm{OSi}$ : 324.1909, found: 324.1905.

5.5.4. trans-1-(Dimethylphenylsilyl)-2-(2-phenoxyphenyl)-cyclohexane 7e. $(1 S, 2 R)-7 e: 20 \%$, viscous oil,
$[\alpha]_{\mathrm{D}}^{20}+25(c 1.0, \mathrm{EtOH}) ;(1 R, 2 S)-7 \mathrm{e}: 25 \%$, viscous oil, $[\alpha]_{\mathrm{D}}^{20}-21(c 1.0, \mathrm{EtOH})$.

${ }^{1} \mathrm{H}$ NMR $\left(\mathrm{CDCl}_{3}, 200 \mathrm{MHz}\right) \delta:-0.13(\mathrm{~s}, 3 \mathrm{H}), 0.18(\mathrm{~s}, 3 \mathrm{H})$, 0.99-1.70 (m, 6H), 1.72-2.0 (m, 3H), 3.0-3.2 (br m, 1H), $6.76(\mathrm{~d}, 1 \mathrm{H}, J=7.9 \mathrm{~Hz}), 6.92-7.12(\mathrm{~m}, 3 \mathrm{H}), 7.22-7.44(\mathrm{~m}$, $10 \mathrm{H}) ;{ }^{13} \mathrm{C} \mathrm{NMR}\left(\mathrm{CDCl}_{3}, 50 \mathrm{MHz}\right) \delta:-4.77,-2.52$, $27.10,27.46,28.62,29.05,33.68,40.05,118.5(2 \mathrm{C})$, 122.82, 126.81 (2C), 127.47 (2C), 127.69, 127.59, 128.38 (2C), 133.6, 133.83 (2C), 133.98, 140.1, 154.30, 156.09; IR (film): 769, 812, 834, 1064, 1112, 1227, 1246, 1426, 1443, 1482, 1596, 2847, 2922, 3020, $3066 \mathrm{~cm}^{-1}$; MS (+CI) $\mathrm{m} / \mathrm{z}(\%): 135.1$ (11), 169.2 (13), 271.1 (100), 309.5 (4), 371.3 (9), $387.7\left(3, \mathrm{M}^{+}+1\right)$; HRMS (CI) calculated for $\mathrm{C}_{26} \mathrm{H}_{30} \mathrm{OSi}$ : 386.2066, found: 386.2096.

5.5.5. trans-1-(Dimethylphenylsilyl)-6,6-dimethyl-2-(1naphthyl)-cyclohexane 7f. $(1 R, 2 R)-7 f$ : $31 \%$, viscous oil, $[\alpha]_{\mathrm{D}}^{20}+69.9(c 0.83, \mathrm{EtOH}) ;(1 S, 2 S)-7 \mathbf{f}, 27 \%$, viscous oil, $[\alpha]_{\mathrm{D}}^{20}-68.1(c 0.83, \mathrm{EtOH})$.

${ }^{1} \mathrm{H} \mathrm{NMR}\left(\mathrm{CDCl}_{3}, 200 \mathrm{MHz}\right) \delta: 0.44(\mathrm{~s}, 3 \mathrm{H}), 0.54(\mathrm{~s}, 3 \mathrm{H})$, $1.05(\mathrm{~s}, 3 \mathrm{H}), 1.33(\mathrm{~s}, 3 \mathrm{H}), 1.20-1.45(\mathrm{~m}, 2 \mathrm{H}), 1.63-1.98$ (m, 4H), 2.14-2.29 (m, 1H), 3.30-3.48 (m, 1H), 7.27-7.68 (m, 10H), 7.79-7.84 (dd, 1H, J=6.6, 1.6 Hz), $7.95(\mathrm{~d}, 1 \mathrm{H}$, $J=6.2 \mathrm{~Hz}) ;{ }^{13} \mathrm{C} \mathrm{NMR}\left(\mathrm{CDCl}_{3}, 50 \mathrm{MHz}\right) \delta: 0.3,0.86$, $29.22,30.32,30.86,32.44,33.58,35.81,36.20,38.26$, $122.40,123.19,125.18,125.57$ (2C), 126.26, 127.74 (2C), $128.53,128.95,131.45,133.79$ (2C), 133.80, 141.10, 143.51; IR (film): 776, 813, 831, 1112, 1249, 1427, 1509, 1597, 2864, 2948, 3047, $3067 \mathrm{~cm}^{-1}$; MS (+CI) $\mathrm{m} / \mathrm{z}(\%)$ : 76.8 (17), 135.0 (16), 136.8 (19), 194.8 (100), 271.1 (47), $357.1(4), 372.7\left(2, \mathrm{M}^{+}\right), 373.2\left(3, \mathrm{M}^{+}+1\right)$; HRMS (CI) calculated for $\mathrm{C}_{26} \mathrm{H}_{32} \mathrm{Si}$ : 372.2273 , found: 372.2265 .

5.5.6. trans-1-(Dimethylphenylsilyl)-2-(1-naphthyl)cyclopentane $\mathbf{7 g} \cdot(1 S, 2 R)-\mathbf{7 g}$ : $32 \%$, viscous oil, $[\alpha]_{\mathrm{D}}^{20}+15$ (c $0.2, \mathrm{EtOH}) ;(1 R, 2 S)-7 \mathrm{~g}: 32 \%$, viscous oil, $[\alpha]_{\mathrm{D}}^{20}-26$ (c $0.39, \mathrm{EtOH})$.

${ }^{1} \mathrm{H} \mathrm{NMR}\left(\mathrm{CDCl}_{3}, 500 \mathrm{MHz}\right) \delta: 0.03(\mathrm{~s}, 3 \mathrm{H}), 0.07$ (s, 3H), $1.60(\mathrm{~m}, 1 \mathrm{H}), 1.69(\mathrm{~m}, 2 \mathrm{H}), 1.80(\mathrm{~m}, 2 \mathrm{H}), 2.07(\mathrm{~m}, 1 \mathrm{H})$, $2.20(\mathrm{~m}, 1 \mathrm{H}), 3.67(\mathrm{td}, 1 \mathrm{H}, J=8.6,8.6,10.2 \mathrm{~Hz}), 7.25(\mathrm{~m}$, 2H), $7.30(\mathrm{~m}, 1 \mathrm{H}), 7.36(\mathrm{~m}, 2 \mathrm{H}), 7.37(\mathrm{~m}, 1 \mathrm{H}), 7.43(\mathrm{~m}$, 1H), $7.46(\mathrm{~m}, 2 \mathrm{H}), 7.66(\mathrm{dd}, 1 \mathrm{H}, J=7.4,1.6 \mathrm{~Hz}), 7.84$ $(\mathrm{m}, 1 \mathrm{H}), 8.08(\mathrm{~m}, 1 \mathrm{H}) ;{ }^{13} \mathrm{C} \mathrm{NMR}\left(\mathrm{CDCl}_{3}, 125 \mathrm{MHz}\right) \delta$ : $-4.54,-3.56,26.07,28.95,32.54,37.86,42.94,123.37$, 123.38, 125.01, 125.30 (2C), 126.03, 127.36 (2C), 128.56, 128.70, 131.85, 133.82 (3C), 138.38, 142.36; IR (film): 793, 830, 1060, 1118, 1245, 1427, 1591, 2865, 2955, 3049, $3068 \mathrm{~cm}^{-1}$; MS (EI, $\left.70 \mathrm{eV}\right) \mathrm{m} / \mathrm{z}(\%): 56.8$ (75), 135.0 (14), 151.0 (37), 193.0 (58), 271.0 (100), 330.1 (5, $\mathrm{M}^{+}$); HRMS (EI) calculated for $\mathrm{C}_{23} \mathrm{H}_{26} \mathrm{Si}$ : 330.1804 , found: 330.1797 .

5.5.7. trans-1-(Diethylphenylsilyl)-2-phenyl-cyclohexane 7h. $(1 R, 2 S)-7 \mathbf{h}: 35 \%$, viscous oil, $[\alpha]_{\mathrm{D}}^{20}+56(c 0.8$, EtOH $)$; $(1 S, 2 R)-7 \mathbf{h}: 31 \%$, viscous oil, $[\alpha]_{\mathrm{D}}^{20}-59(c 0.8, \mathrm{EtOH})$.

${ }^{1} \mathrm{H}$ NMR $\left(\mathrm{CDCl}_{3}, 200 \mathrm{MHz}\right) \delta: 0.12(\mathrm{~m}, 1 \mathrm{H}), 0.27(\mathrm{~m}, 1 \mathrm{H})$, $0.53(\mathrm{q}, 2 \mathrm{H}, J=7.6 \mathrm{~Hz}), 0.77(\mathrm{t}, 6 \mathrm{H}, J=7.7 \mathrm{~Hz}), 1.10-1.48$ $(\mathrm{m}, 5 \mathrm{H}), 1.65-1.90(\mathrm{~m}, 4 \mathrm{H}), 2.21-2.40(\mathrm{~m}, 1 \mathrm{H}), 7.03-7.07$ $(\mathrm{dd}, 2 \mathrm{H}, J=7.9,2.1 \mathrm{~Hz}), 7.14-7.31(\mathrm{~m}, 8 \mathrm{H}) ;{ }^{13} \mathrm{C} \mathrm{NMR}$ 
$\left(\mathrm{CDCl}_{3}, 50 \mathrm{MHz}\right) \delta: 1.76,2.34,7.28,7.52,26.97,27.62$, $28.48,28.92,38.28,46.54,126.06,127.33$ (2C), 127.87 (2C), 128.01 (2C), 128.41, 134.69 (2C), 136.68, 147.62; IR (film): 734, 757, 1007, 1057, 1107, 1235, 1427, 1444, 1490, 1600, 2873, 2919, 3025, $3067 \mathrm{~cm}^{-1}$; MS (+CI) $\mathrm{m} / \mathrm{z}$ (\%): 163.0 (24), 164.3 (4), 245.1 (97), 293.2 (100), 323.3 $\left(1, \mathrm{M}^{+}+1\right)$; HRMS (CI) calculated for $\mathrm{C}_{22} \mathrm{H}_{30} \mathrm{Si}$ : 322.2116, found: 322.2120 .

5.5.8. trans-2-Benzyl-1-(dimethylphenylsilyl)-cyclohexane 8a. $(1 S, 2 R)-8 \mathbf{a}: 54 \%$, viscous oil, $[\alpha]_{\mathrm{D}}^{20}+11(c 0.48$, $\mathrm{EtOH}) ;(1 R, 2 S)-8 \mathrm{a}: 85 \%$, viscous oil, $[\alpha]_{\mathrm{D}}{ }^{20}-14.3(c$ 0.42 , EtOH).

${ }^{1} \mathrm{H}$ NMR $\left(\mathrm{CDCl}_{3}, 500 \mathrm{MHz}\right) \delta: 0.38(\mathrm{~s}, 3 \mathrm{H}), 0.42(\mathrm{~s}, 3 \mathrm{H})$, $0.86(\mathrm{td}, 1 \mathrm{H}, J=11.5,10.5,3.1 \mathrm{~Hz}), 0.88(\mathrm{~m}, 1 \mathrm{H}), 1.10(\mathrm{qt}$, $1 \mathrm{H}, J=11.5,3.3 \mathrm{~Hz}$ ), $1.22(\mathrm{qt}, 1 \mathrm{H}, J=11.5,3.1 \mathrm{~Hz}), 1.28$ $(\mathrm{qd}, 1 \mathrm{H}, J=11.5,3.1 \mathrm{~Hz}), 1.52(\mathrm{dq}, 1 \mathrm{H}, J=12.5,3.6$, $3.3 \mathrm{~Hz}), 1.55$ (qt, $1 \mathrm{H}, J=10.5,3.6 \mathrm{~Hz}), 1.62(\mathrm{~m}, 1 \mathrm{H}), 1.69$ $(\mathrm{m}, 1 \mathrm{H}), 1.81(\mathrm{dq}, 1 \mathrm{H}, J=11.5,3.1 \mathrm{~Hz}), 2.00(\mathrm{dd}, 1 \mathrm{H}$, $J=13.4,10.5 \mathrm{~Hz}), 2.94(\mathrm{dd}, 1 \mathrm{H}, J=13.4,3.6 \mathrm{~Hz}), 6.94(\mathrm{~m}$, 2H), $7.14(\mathrm{~m}, 1 \mathrm{H}), 7.21(\mathrm{~m}, 2 \mathrm{H}), 7.37(\mathrm{~m}, 3 \mathrm{H}), 7.58(\mathrm{~m}$, $2 \mathrm{H}) ;{ }^{13} \mathrm{C} \mathrm{NMR}\left(\mathrm{CDCl}_{3}, 125 \mathrm{MHz}\right) \delta:-3.06,-2.24$, $25.88,27.69,28.26,30.80,32.63,40.85,43.26,125.37$, 127.63 (2C), 127.86 (2C), 128.53, 128.90 (2C), 133.67 (2C), 139.81, 141.48; IR (film): 699, 733, 768, 831, 1051, 1063, 1114, 1251, 1427, 1588, 1603, 2850, 2927, 3024, $3067 \mathrm{~cm}^{-1}$; MS (+CI) $\mathrm{m} / \mathrm{z}(\%): 135.0$ (18), 217.0 (10), 231.0 (100), 293.1 (39), 307.1 (5), $309.2\left(2, \mathrm{M}^{+}+1\right)$; HRMS (CI) calculated for $\mathrm{C}_{21} \mathrm{H}_{28} \mathrm{Si}$ : 308.1960, found: 308.1955 .

5.5.9. trans-1-(Dimethylphenylsilyl)-2-(2-naphthylmethyl)-cyclohexane $8 \mathbf{b}$. $(1 S, 2 R)-8 \mathbf{b}: 34 \%$, viscous oil, $[\alpha]_{\mathrm{D}}^{20}+33(c 0.41, \mathrm{EtOH}) ;(1 R, 2 S)-8 \mathbf{b}: 38 \%$, viscous oil, $[\alpha]_{\mathrm{D}}^{20}-35(c 0.4, \mathrm{EtOH})$.

${ }^{1} \mathrm{H} \quad \mathrm{NMR} \quad\left(\mathrm{CDCl}_{3}, \quad 500 \mathrm{MHz}\right) \quad \delta:{ }^{13} \mathrm{C} \quad \mathrm{NMR} \quad\left(\mathrm{CDCl}_{3}\right.$, $125 \mathrm{MHz}):-2.95,-2.14,25.84,27.69,28.32,30.85$, $32.73,40.82,43.35,124.83,125.62,127.05,127.23$, $127.33,127.42,127.62,127.7$ (2C), 128.61, 131.74, 133.31, 133.75 (2C), 139.01, 139.77; IR (film): 831, 1112, 1248, 1426, 1444, 1600, 2849, 2925, $3049 \mathrm{~cm}^{-1}$; MS (+CI) $\mathrm{m} / \mathrm{z}(\%): 127.1$ (23), 135.0 (13), 141.1 (24), 281.1 (100), 343.2 (16), $358.0\left(4, \mathrm{M}^{+}\right), 359.1\left(2, \mathrm{M}^{+}+1\right)$; HRMS (CI) calculated for $\mathrm{C}_{25} \mathrm{H}_{30} \mathrm{Si}$ : 358.2117 , found: 358.2112 .

5.5.10. trans-1-(Dimethylphenylsilyl)-2-(1-naphthylmethyl)-cyclohexane 8c. $(1 S, 2 R)-8 c$ : $31 \%$, viscous oil, $[\alpha]_{\mathrm{D}}^{20}+45(c 0.75, \mathrm{EtOH}) ;(1 R, 2 S)-8 \mathrm{c}: 33 \%$, viscous oil, $[\alpha]_{\mathrm{D}}^{20}-44(c 0.7, \mathrm{EtOH})$.

${ }^{1} \mathrm{H}$ NMR $\left(\mathrm{CDCl}_{3}, 500 \mathrm{MHz}\right) \delta: 0.44(\mathrm{~s}, 3 \mathrm{H}), 0.48(\mathrm{~s}, 3 \mathrm{H})$, $0.93(\mathrm{~m}, 2 \mathrm{H}), 1.07$ (qt, $1 \mathrm{H}, J=12.3,3.6 \mathrm{~Hz}), 1.26(\mathrm{~m}, 2 \mathrm{H})$, $1.42(\mathrm{~m}, 1 \mathrm{H}), 1.59(\mathrm{~m}, 1 \mathrm{H}), 1.69(\mathrm{~m}, 1 \mathrm{H}), 1.84(\mathrm{~m}, 1 \mathrm{H})$, $1.93(\mathrm{~m}, 1 \mathrm{H}), 2.51(\mathrm{dd}, 1 \mathrm{H}, J=11.3,14.4 \mathrm{~Hz}), 3.37(\mathrm{dd}$, $1 \mathrm{H}, J=14.4,4.1 \mathrm{~Hz}), 7.13(\mathrm{~d}, 1 \mathrm{H}, J=7.0 \mathrm{~Hz}), 7.29(\mathrm{~m}$, $1 \mathrm{H}), 7.34(\mathrm{t}, 1 \mathrm{H}, J=7.3 \mathrm{~Hz}), 7.42(\mathrm{~m}, 4 \mathrm{H}), 7.47(\mathrm{~d}, 1 \mathrm{H}$, $J=8.5 \mathrm{~Hz}), 7.65(\mathrm{dd}, 2 \mathrm{H}, J=7.3,1.6 \mathrm{~Hz}), 7.67(\mathrm{~d}, 1 \mathrm{H}$, $J=8.0 \mathrm{~Hz}), 7.80(\mathrm{~d}, 1 \mathrm{H}, J=8.1 \mathrm{~Hz}) ;{ }^{13} \mathrm{C} \mathrm{NMR}\left(\mathrm{CDCl}_{3}\right.$, $125 \mathrm{MHz})$ : $-2.35,-1.83,25.75,27.71,28.26,32.30$, $32.89,39.57,40.37,124.17,125.01,125.02,125.27$, $126.19,127.07,127.82$ (2C), 128.37, 128.71, 132.08,
133.60, 133.85 (2C), 137.37, 139.57; IR (film): 767, 784, $809,832,1112,1247,1426,1444,1508,1595,2849$, 2927, 3045, $3066 \mathrm{~cm}^{-1}$; MS (+CI) m/z (\%): 99.0 (100), 135.0 (10), 141.1 (11), 217.0 (5), 281.1 (12), 343.2 (4), $358.4\left(1, \mathrm{M}^{+}\right)$; HRMS (CI) calculated for $\mathrm{C}_{25} \mathrm{H}_{30} \mathrm{Si}$ : 358.2117, found: 358.2120 .

\subsection{Synthesis of ENP silyl dithioketals 10a,b}

General procedure: to a $25 \mathrm{~mL}$ two-neck flask equipped with a tube of blue silica gel and stirring bar, were added $0.3 \mathrm{~g}$ (0.972 mmol, 1 equiv) (-)-4a, $0.101 \mathrm{~mL}$ (114 mg, $1.22 \mathrm{mmol}, 1.25$ equiv) 1,2-ethanedithiol and $8 \mathrm{~mL}$ dry $\mathrm{CH}_{2} \mathrm{Cl}_{2}$. The solution was cooled to $-40{ }^{\circ} \mathrm{C}$ and $0.032 \mathrm{~mL}$ ( $55 \mathrm{mg}, 0.29$ mmol, 0.3 equiv) $\mathrm{TiCl}_{4}$ was added under argon. The reaction mixture was warmed to room temperature and stirred for additional $5 \mathrm{~h}$ before the reaction was quenched with $3 \mathrm{~mL}$ saturated $\mathrm{NaHCO}_{3}$ solution. The mixture was extracted three times with $\mathrm{CH}_{2} \mathrm{Cl}_{2}$. The organic phases were dried, filtered and evaporated in vacuum. Purification of the residue by flash chromatography $\left(\mathrm{EP} / \mathrm{Et}_{2} \mathrm{O}=30 / 1\right.$, $\left.R_{f}=0.33\right)$ gave $0.62 \mathrm{~g}(90 \%$, yield) product $(-)-\mathbf{1 0 a}$ as viscous oil.

5.6.1. Compound 10a. $[\alpha]_{\mathrm{D}}^{20}+22.3(c$ 1.0, EtOH), viscous oil, $88 \%$; $[\alpha]_{\mathrm{D}}^{20}-18$ (c 1.0, EtOH), viscous oil, $90 \%$.

${ }^{1} \mathrm{H} \mathrm{NMR}\left(\mathrm{CDCl}_{3}, 500 \mathrm{MHz}\right) \delta:-0.11(\mathrm{~s}, 3 \mathrm{H}),-0.06(\mathrm{~s}, 3 \mathrm{H})$, $1.66(\mathrm{~m}, 1 \mathrm{H}), 1.88(\mathrm{~m}, 3 \mathrm{H}), 2.03(\mathrm{~m}, 1 \mathrm{H}), 2.15(\mathrm{~m}, 1 \mathrm{H})$, $2.65(\mathrm{~m}, 1 \mathrm{H}), 3.36(\mathrm{~d}, 1 \mathrm{H}, J=3.1 \mathrm{~Hz}), 3.21-3.33(\mathrm{~m}, 4 \mathrm{H})$, 7.13-7.43 (m, $10 \mathrm{H}) ;{ }^{13} \mathrm{C} \mathrm{NMR}\left(\mathrm{CDCl}_{3}, 125 \mathrm{MHz}\right) \delta$ : $-5.05,-4.49,20.40,26.29,30.25,38.05,38.48,39.14$, $55.55,74.44,126.92,127.37$ (2C), 127.54 (2C), 128.52, 130.69 (2C), 133.70 (2C), 138.10, 142.46; IR (film): 770, $812,836,1111,1248,1245,1426,1494,1599,2854$, 2923, 3024, $3066 \mathrm{~cm}^{-1}$; MS (+CI) m/z (\%): 124.8 (100), 135.1 (10), 248.9 (48), 369.0 (13), $385.2\left(16, \mathrm{M}^{+}+1\right)$; HRMS (CI) calculated for $\mathrm{C}_{22} \mathrm{H}_{28} \mathrm{~S}_{2} \mathrm{Si}$ : 384.1401, found: 384.1396.

5.6.2. Compound 10b. $[\alpha]_{\mathrm{D}}^{20}+28(c 0.8$, EtOH), viscous oil, $47 \% ;[\alpha]_{\mathrm{D}}^{20}-25$ (c $\left.0.8, \mathrm{EtOH}\right)$, viscous oil, $50 \%$.

${ }^{1} \mathrm{H} \mathrm{NMR}\left(\mathrm{CDCl}_{3}, 200 \mathrm{MHz}\right) \delta:-0.26(\mathrm{~s}, 3 \mathrm{H}),-0.25(\mathrm{~s}, 3 \mathrm{H})$, $1.26-1.34(\mathrm{~m}, 1 \mathrm{H}), 1.60-1.72(\mathrm{~m}, 2 \mathrm{H}), 2.02-2.17(\mathrm{~m}, 2 \mathrm{H})$, 2.68-2.99 (m, 2H), 3.09-3.16 (m, 2H), 3.34-3.42 (m, 2H), $4.42(\mathrm{~d}, 1 \mathrm{H}, J=3.2 \mathrm{~Hz}), 7.05(\mathrm{dd}, 1 \mathrm{H}, J=7.9,1.5 \mathrm{~Hz})$, 7.11-7.45 (m, 7H), 7.72-7.90 (m, 4H); ${ }^{13} \mathrm{C} \mathrm{NMR}\left(\mathrm{CDCl}_{3}\right.$, $50 \mathrm{MHz}) \delta:-4.26,-4.12,20.88,26.51,30.43,38.00$, $38.63, \quad 39.47,45.87,74.85, \quad 124.48,124.87,124.94$, $125.40,126.69,127.47$ (2C), 127.80, 128.51, 128.71, $133.49,133.65$ (2C), 133.82, 138.18, 139.49; IR (film) in $\mathrm{cm}^{-1}:$ 779, 797, 834, 1117, 1255, 1427, 1488, 1597, 2859, 2928, 3049, 3067; MS (+CI) m/z (\%): 58.9 (100), 74.9 (60), 135.0 (17), 136.8 (19), 207.1 (64), 299.2 (37), 419.2 (10), $434.2\left(10, \mathrm{M}^{+}\right), 435.3\left(10, \mathrm{M}^{+}+1\right)$.

\subsection{Synthesis of cis-2-arylmethyl-1-dialkylphenylsilyl cycloalkanones $9 a, b$}

General procedure: to a solution of $0.45 \mathrm{~g}(1.17 \mathrm{mmol}$, 1.0 equiv) (-)-10a in $5 \mathrm{~mL} \mathrm{MeOH}$, an excess of Raney nickel was added (10-fold). The reaction mixture was stirred 
at room temperature for $10 \mathrm{~h}$ (TLC check). The mixture was filtered through a pad of Celite and washed with $\mathrm{CH}_{2} \mathrm{Cl}_{2}$. The filtrate was extracted with $3 \times 5 \mathrm{~mL} \mathrm{CH}_{2} \mathrm{Cl}_{2}$. The organic layer was dried, filtered and concentrated. Flash chromatography of the residue $\left(R_{f}=0.45\right.$, hexane) afforded $0.21 \mathrm{~g}$ $(60 \%)$ product $(-)-9 a$ as viscous oil.

5.7.1. cis-1-(Dimethylphenylsilyl)-2-phenyl-cyclohexane 11a. (+)-9a: $[\alpha]_{\mathrm{D}}^{20}+24(c 1.0$, EtOH), viscous oil, $58 \%$; (-)-9a: $[\alpha]_{\mathrm{D}}^{20}-25.6$ (c 1.0, EtOH), viscous oil, $60 \%$.

${ }^{1} \mathrm{H}$ NMR $\left(\mathrm{CDCl}_{3}, 200 \mathrm{MHz}\right) \delta:-0.20(\mathrm{~s}, 3 \mathrm{H}), 0.09$ (s, 3H), 1.42-1.70 (m, 5H), 1.71-1.81 (m, 4H), 2.99-3.04 (m, 1H), 7.14-7.26 (m, 5H), 7.27-7.48 (m, 5H); ${ }^{13} \mathrm{C} \mathrm{NMR}\left(\mathrm{CDCl}_{3}\right.$, $50 \mathrm{MHz}) \delta$ : $-2.40,-1.87,24.54,25.85,27.31,30.94$, $32.17,43.83,125.86,127.39$ (2C), 127.83 (2C), 128.18 (2C), 128.37, 133.80 (2C), 139.99, 146.41; IR (film) in: $814,834,1112,1249,1427,1446,1600,2848,2923,3025$, $3066 \mathrm{~cm}^{-1}, \mathrm{MS}(+\mathrm{CI}) \mathrm{m} / \mathrm{z}(\%): 135.1$ (4), 217.2 (30), 279.2 (100), 293.2 (28), 294.0 (24, 295.1 (8, M+1); HRMS (CI) calculated for $\mathrm{C}_{20} \mathrm{H}_{26} \mathrm{Si}$ : 294.1803, found: 294.1796.

5.7.2. cis-1-(Dimethylphenylsilyl)-2-(1-naphthyl)-cyclohexane 9b. $(+)-9 \mathrm{~b}:[\alpha]_{\mathrm{D}}^{20}+30(c 0.6, \mathrm{EtOH})$, viscous oil, 19\%; (-)-9b: $[\alpha]_{\mathrm{D}}^{20}-31$ (c $\left.0.5, \mathrm{EtOH}\right)$, viscous oil, $20 \%$.

${ }^{1} \mathrm{H}$ NMR $\left(\mathrm{CDCl}_{3}, 200 \mathrm{MHz}\right) \delta:-0.41(\mathrm{~s}, 3 \mathrm{H}), 0.12(\mathrm{~s}, 3 \mathrm{H})$, $1.40-1.70(\mathrm{~m}, 4 \mathrm{H}), 1.71-2.10(\mathrm{~m}, 5 \mathrm{H}), 3.70-3.80(\mathrm{~m}, 1 \mathrm{H})$, 7.11-7.26 (m, 4H), 7.34-7.53 (m, 5H), 7.63-7.68 (dd, $1 \mathrm{H}, J=6.0,1.9 \mathrm{~Hz}), 7.78-7.80(\mathrm{~m}, 1 \mathrm{H}), 8.04(\mathrm{~d}, 1 \mathrm{H}$, $J=7.8 \mathrm{~Hz}) ;{ }^{13} \mathrm{C} \mathrm{NMR}\left(\mathrm{CDCl}_{3}, 50 \mathrm{MHz}\right) \delta:-4.10,-0.87$, $25.48,26.85,28.00,30.98,32.27,42.81,122.92,124.86$, $125.00,125.46,125.84,126.48,127.48$ (2C), 128.88, $128.98,131.05,133.86$ (2C), 133.99, 139.04, 143.78; IR (film): 776, 817, 834, 1112, 1248, 1444, 1596, 2848, 2924, 3046, $3067 \mathrm{~cm}^{-1}$; MS (+CI) $\mathrm{m} / \mathrm{z}(\%): 135.1$ (3), 267.2 (86), 295.2 (14), 329.2 (72), $344.2\left(100, \mathrm{M}^{+}\right), 345.3$ (28, $\left.\mathrm{M}^{+}+1\right)$; HRMS (CI) calculated for $\mathrm{C}_{24} \mathrm{H}_{28} \mathrm{Si}$ : 344.1960, found: 344.1954 .

\subsection{General procedure for in situ preparation of sily- lated triflimides and catalysed Diels-Alder reactions}

To a $25 \mathrm{ml}$ two-neck flask equipped with a tube of silica gel and a stirring bar, under argon were added $15 \mathrm{~mol} \%$ ENP dialkylphenylsilyl-cycloalkane (1 equiv) and enough dry $\mathrm{CH}_{2} \mathrm{Cl}_{2}$ [solvent/diene=3:1 (v/v)]. A solution of bis(trifluoromethanesulfonyl)imide $(10 \mathrm{~mol} \%)$ in $\mathrm{CH}_{2} \mathrm{Cl}_{2}$ was added at room temperature and the reaction continued for 2-3 h. 2,6-Di(tert-butyl)-4-methylpyridine (20 mol \%, DTBMP, dissolved in $\mathrm{CH}_{2} \mathrm{Cl}_{2}$ ) was added and the mixture cooled to the temperature mentioned in the table. Methyl acrylate (1 equiv) was added and followed by cyclopentadiene (4 equiv) $15 \mathrm{~min}$ later. The reaction mixture was stirred for $1.5 \mathrm{~h}$ (checked by ${ }^{1} \mathrm{H} \mathrm{NMR}$ ) and quenched with $3 \mathrm{~mL}$ saturated $\mathrm{NaHCO}_{3}$ aqueous solution. The mixture was extracted with $3 \times 10 \mathrm{~mL} \mathrm{CH}_{2} \mathrm{Cl}_{2}$, and the combined organic phases were dried, filtered and evaporated. The products were isolated by flash chromatography (yields, see Table 5). Enantiomeric excesses were determined by chiral GC. Conversions and ratio of endo/exo were measured by ${ }^{1} \mathrm{H}$ NMR on the crude product (endo/ exo also by chiral GC).

\section{Acknowledgements}

This work was generously supported by Rhodia and the Université catholique de Louvain. We thank Dr. R. Touillaux for NMR analyses. G.D. is research associate of the FRS-FNRS, Brussels. We wish also to acknowledge financial support from the Belgian Program on Interuniversity Poles of Attraction (IAP no P5/33). We thank Dr. Loic Guillonneau for his help with the preparation of the manuscript.

\section{References and notes}

1. (a) Ghosez, L. Stereocontrolled Organic Synthesis; Trost, B., Ed.; Blackwell Scientific: Oxford, 1994; p 193; (b) Mbiya, K. Dissertation, Université catholique de Louvain, Belgium, 1994.

2. (a) Jnoff, E.; Ghosez, L. J. Am. Chem. Soc. 1999, 121, 26172618; (b) Ntirampebura, D; Ghosez, L. Tetrahedron Lett. 1999, 40, 7079-7082; Synthesis 2002, 2043-2052; (c) Johnson, J. S.; Evans, D. A. Acc. Chem. Res. 2000, 33, 325-335.

3. Happaerts, T. Dissertation, Université catholique de Louvain, Belgium, 2002.

4. (a) Lamy-Schelkens, H.; Giomi, D.; Ghosez, L. Tetrahedron Lett. 1989, 30, 5887-5890; (b) Lamy-Schelkens, H.; Ghosez, L. Tetrahedron Lett. 1989, 30, 5891-5894.

5. Mathieu, B.; Ghosez, L. Tetrahedron Lett. 1997, 38, 54975500.

6. Ishii, A.; Kotera, O.; Saeki, T.; Mikami, K. Synlett 1997, 1145.

7. Mathieu, B.; de Fays, L.; Ghosez, L. Tetrahedron Lett. 2000, 41, 9561-9564.

8. Examples: Berry, M. B.; Griffiths, R. J.; Yufit, D. S.; Steel, P. G. Chem. Commun. 1998, 2155-2156; Kuhnert, N.; Peverley, J.; Robertson, J. Tetrahedron Lett. 1998, 39, 3215-3216; Othman, R. B.; Bousquet, T.; Othman, M.; Dalla, V. Org. Lett. 2005, 7, 5335-5337; Hara, K.; Akiyama, R.; Sawamura, M. Org. Lett. 2005, 7, 5621-5623; Ishihara, K.; Hiraiwa, Y.; Yamamoto, H. Synlett 2001, 1851-1854.

9. Desmurs, J. R.; Ghosez, L.; Martins, J.; Deforth, T.; Mignani, G. J. Organomet. Chem. 2002, 646, 171-178.

10. Johannsen, M.; Jorgensen, K. A.; Helmchen, G. A. J. Am. Chem. Soc. 1998, 120, 7637-7638.

11. (a) Shirakawa, S.; Lombardi, P. J.; Leighton, J. L. J. Am. Chem. Soc. 2005, 127, 9974-9975; (b) Kubota, K.; Hamblett, C. L.; Wang, X.; Leighton, J. L. Tetrahedron 2006, 62, 11397-11401.

12. (a) Hawkins, J. M.; Loren, S. J. Am. Chem. Soc. 1991, 113, 7794-7795; (b) Hawkins, J. M.; Loren, S. J. Am. Chem. Soc. 1994, 116, 1657-1660; (c) Hawkins, J. M.; Nambu, N.; Loren, S. Org. Lett. 2003, 5, 4293-4295.

13. Honma, T.; Nakajo, M.; Mizugaki, T.; Ebitani, K.; Kaneda, K. Tetrahedron Lett. 2002, 43, 6229-6232.

14. (a) Corey, E. J.; Melvin, L. S.; Haslanger, M. F. Tetrahedron Lett. 1975, 36, 3117-3120; (b) Fuchs, P. L. J. Org. Chem. 1976, 41, 2935-2937; (c) Stork, G.; Ponaras, A. A. J. Org. Chem. 1976, 41, 2937-2939; (d) Wender, P. A.; Erhardt, J. M.; Letendre, L. J. J. Am. Chem. Soc. 1981, 103, 2114-2116.

15. Solladie, G.; Hutt, J. J. Org. Chem. 1987, 52, 3560-3566.

16. Jean, K. Y.; Cava, M. P. J. Org. Chem. 1983, 48, 1449-1451.

17. Rivaka, B. S.; David, G. J. Chem. Soc. 1957, 1289.

18. (a) Ager, D. J.; Fleming, I. J. Chem. Soc., Chem. Commun. 1978, 177-178; (b) Ager, D. J.; Fleming, I.; Patel, S. K. 
J. Chem. Soc., Perkin Trans. 1 1981, 2520-2526; (c) Mathieu, B. Ph.D. Dissertation, Université catholique de Louvain, Belgium, 2001.

19. Hatzigrigoriou, E.; Wartski, L.; Seyden-Penne, J.; Toromanoff, E. Tetrahedron 1985, 41, 5045-5050.

20. Pretsch, E.; Clercq, T.; Seibl, N.; Simon, W. Table of Spectral Data For Structure Determination of Organic Compounds, 2nd ed.; Springer: Berlin, Heidelberg, 1989.

21. Engel, W.; Fleming, I.; Smithers, R. H. J. Chem. Soc., Perkin Trans. 1 1986, 1637-1641.

22. Crystallographic data have been deposited with the Cambridge Crystallographic Data Centre and allocated deposition numbers CCDC 642125-642127.
23. Kim, S.; Chang, H. O.; Ko, J. S.; Ahn, K. H.; Kim, Y. J. J. Org. Chem. 1985, 50, 1927-1932.

24. Kumar, V.; Dev, S. Tetrahedron Lett. 1983, 24, 1289-1292.

25. Rao Narasimha, P. J. Org. Chem. 1971, 36, 2426-2434.

26. Mathieu, B.; Ghosez, L. Tetrahedron 2002, 58, 8219-8226.

27. Loncharich, R. J.; Schwarz, T. R.; Houk, K. N. J. Am. Chem. Soc. 1987, 109, 5502-5503.

28. DFT (density functional theory) B3LYP calculations. The function basis set is the double zeta $6-31 \mathrm{G}^{*}$ with one shell of $\mathrm{d}$ orbitals on the second row atoms. All degrees of freedom of the molecular geometry (distances, bond angles, dihedral angles) were completely optimised by minimisation of the partial gradient under the threshold of 15 10-6 Hartree/Bohr-radian. 